\title{
Large-scale phosphoproteome analysis in seedling leaves of Brachypodium distachyon L.
}

\author{
Dong-Wen Lv, Xin Li, Ming Zhang, Ai-Qin Gu, Shou-Min Zhen, Chang Wang, Xiao-Hui Li and Yue-Ming Yan*
}

\begin{abstract}
Background: Protein phosphorylation is one of the most important post-translational modifications involved in the regulation of plant growth and development as well as diverse stress response. As a member of the Poaceae, Brachypodium distachyon $\mathrm{L}$. is a new model plant for wheat and barley as well as several potential biofuel grasses such as switchgrass. Vegetative growth is vital for biomass accumulation of plants, but knowledge regarding the role of protein phosphorylation modification during vegetative growth, especially in biofuel plants, is far from comprehensive.

Results: In this study, we carried out the first large-scale phosphoproteome analysis of seedling leaves in Brachypodium accession $\mathrm{Bd} 21$ using $\mathrm{TiO}_{2}$ microcolumns combined with liquid chromatography-tandem mass spectrometry (LC-MS/MS) and MaxQuant software. A total of 1470 phosphorylation sites in 950 phosphoproteins were identified, and these phosphoproteins were implicated in various molecular functions and basic cellular processes by gene ontology (GO) and Kyoto Encyclopedia of Genes and Genomes (KEGG) pathway analyses. Among the 950 phosphoproteins identified, 127 contained 3 to 8 phosphorylation sites. Conservation analysis showed that $93.4 \%$ of the 950 phosphoproteins had phosphorylation orthologs in other plant species. Motif-X analysis of the phosphorylation sites identified 13 significantly enriched phosphorylation motifs, of which 3 were novel phosphorylation motifs. Meanwhile, there were 91 phosphoproteins with both multiple phosphorylation sites and multiple phosphorylation motifs. In addition, we identified 58 phosphorylated transcription factors across 21 families and found out 6 significantly over-represented transcription factor families (C3H, Trihelix, CAMTA, TALE, MYB_related and CPP). Eighty-four protein kinases (PKs), 8 protein phosphatases (PPs) and 6 CESAs were recognized as phosphoproteins.

Conclusions: Through a large-scale bioinformatics analysis of the phosphorylation data in seedling leaves, a complicated PKs- and PPs- centered network related to rapid vegetative growth was deciphered in B. distachyon. We revealed a MAPK cascade network that might play the crucial roles during the phosphorylation signal transduction in leaf growth and development. The phosphoproteins and phosphosites identified from our study expanded our knowledge of protein phosphorylation modification in plants, especially in monocots.
\end{abstract}

Keywords: Bd21, Leaf, Phosphoproteome, Transcription factors, Phosphorylation motif, Protein kinases

\section{Background}

Protein phosphorylation is one of the common reversible post-translational modifications involved in the regulation of plant growth and diverse processes [1]. Various techniques have been developed for specific enrichment of phosphopeptides, among which $\mathrm{Fe}^{3+}$-IMAC (Immobilized metal affinity chromatography) [2,3], $\mathrm{TiO}_{2}$-MOAC (Metal oxide affinity chromatography) $[4,5]$ and SCX (Strong

\footnotetext{
* Correspondence: yanym@cnu.edu.cn

College of Life Science, Capital Normal University, Beijing 100048, China
}

cation exchange) chromatography [6,7] are the three most common used methods for large-scale phosphoproteome studies. In recent years, through these phosphopeptide enrichment techniques combined with high-accuracy mass spectrometry (MS) and related bioinformatics, many large-scale phosphoproteomic analyses were performed in different plant species, such as Arabidopsis thaliana [8-11], Oryza sativa [10], Medicago truncatula [12,13], Glycine max [11], Zea mays [14,15] and Triticum aestivum [16,17]. However, knowledge of protein phosphorylation modification in other plant species, especially for members of the Poaceae, is far from comprehensive. 
Vegetative growth rapidly increases the photosynthetic capacity and size of plants, and is vital for biomass accumulation, especially in biofuel plants. Brachypodium distachyon L., a member of the Pooideae subfamily and a temperate wild annual grass endemic to the Mediterranean and Middle East [18], has rapidly become a model plant, especially for potential biofuel grasses such as switchgrass (Panicum virgatum L.). It possesses many attractive attributes such as a small diploid genome of $272 \mathrm{Mbp}$, short growth cycle, self-fertility and simple nutrient requirements [19] as well as competence to be efficiently transformed [20].

Leaf expansion is a major aspect of plant vegetative growth. It increases light capture, which powers photosynthesis and thus biomass production. Considerable works have investigated the mechanisms of leaf expansion [21-24], but little is known about post-translational phosphorylation modification of proteins during leaf expansion in the period of rapid vegetative growth. During this period the leaf cell wall undergoes dynamic changes to allow cells to expand, but at the same time cells must maintain the mechanical strength required to resist the forces of turgor pressure [25]. During plant growth and development, leaf cell numbers and size are rapidly increasing and cell walls must adapt to these changes. The components and mechanisms of underlying signaling systems to achieve this process remain largely unknown, but emerging evidences have implicated several receptor-like kinases as regulators of cell wall function [26-30].

Interactions of protein kinases (PKs) with their substrates are to a large extent determined by residues surrounding the phosphorylation sites and the pattern of residues is named as a phosphorylation motif [31,32]. Motif-X analysis of large phosphorylation site data sets can detect significantly enriched phosphorylation motifs and predict the corresponding PKs [33]. Schwartz et al. [34] used Motif-X to determine phosphorylation motifs in yeast, fly, mouse, and man. Trost et al. [35] found eight specific phagosomal phosphorylation motifs induced by IFN- $\gamma$. Bennetzen et al. [36] used Motif-X to analyze phosphorylation sites during DNA damage responses and identified a novel [sxxQ] motif, confirming that Motif-X is a powerful program for revealing significant and novel phosphorylation motifs.

Recently, a large-scale proteomic and phosphoproteomic study of $B$. distachyon leaves under salt stress was performed in our laboratory [37]. In the present study, we focused on phosphorylation modification during seedling leaf growth and development at the omics level for the first time in $B$. distachyon and revealed a complicated phosphorylation signal transduction network during rapid leaf growth and development.

\section{Results and discussion}

Identification of phosphorylation sites and phosphoproteins In this study, a large-scale phosphoproteome analysis in $B$. distachyon seedling leaves was performed to explore the complex protein phosphorylation network of signaling and regulatory events. The strategy used in this study is shown in Figure 1. To increase the number of identified phosphopeptides and eliminate false positives, three biological replicates were used for phosphopeptide enrichment and LC-MS/MS analysis. A total of 1937 phosphopeptides containing 2449 phosphorylation sites were identified (Additional file 1: Table S1). The raw mass spectrometry proteomics data have been deposited to the ProteomeXchange Consortium (http://proteomecentral.proteomexchange.org) via the PRIDE partner repository [38] with the dataset identifier PXD000868. Only the phosphopeptides with unambiguous (class I) phosphorylation sites identified from three biological repeats were used for further analysis. Finally, the 1470 unambiguous phosphorylation sites distributed in 1367 phosphopeptides, corresponding to 950 phosphoproteins, were screened and used for further analysis (Additional file 1: Table S1, sheet A). Among the 1470 unambiguous phosphorylation sites, 1313 Ser, 155 Thr, and 2 Tyr phosphorylation sites were identified, accounting for $89.32 \%$, $10.54 \%$, and $0.14 \%$ of the total sites, respectively (Figure 2, panel A). For the lower identification of phosphorylated tyrosine, there are three possible reasons: first, we didn't perform focused analysis during MS runs in this study, which may lead to the reduced discovery of the low abundant Tyr phosphopeptides; second, the recovery rate of phospho-Tyr peptides could be different because of the enrichment techniques and data processing softwares; third, different organs, development stages or stress treatments can also lead to the variation of the phospho-Tyr

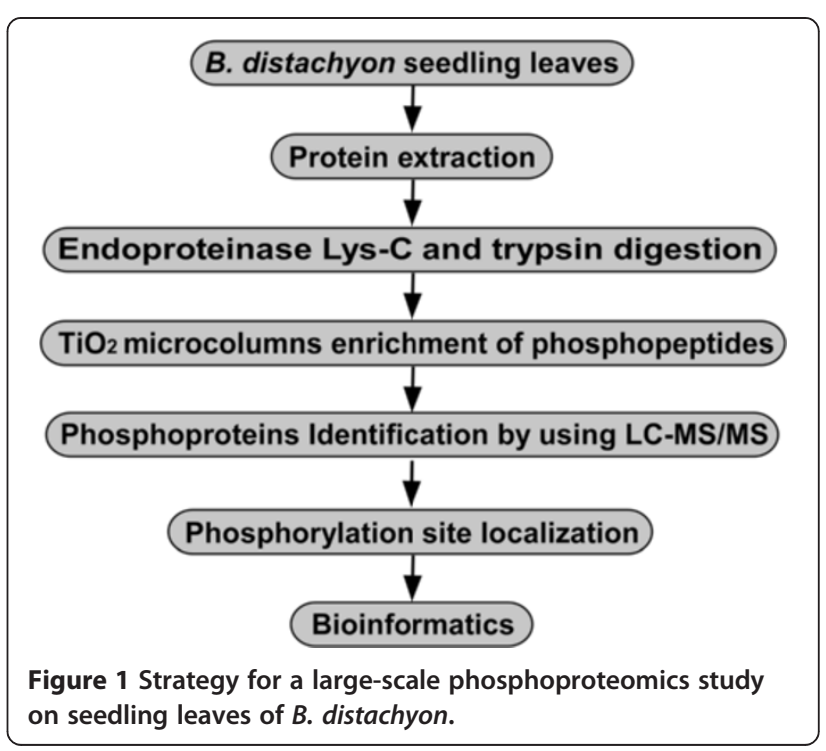




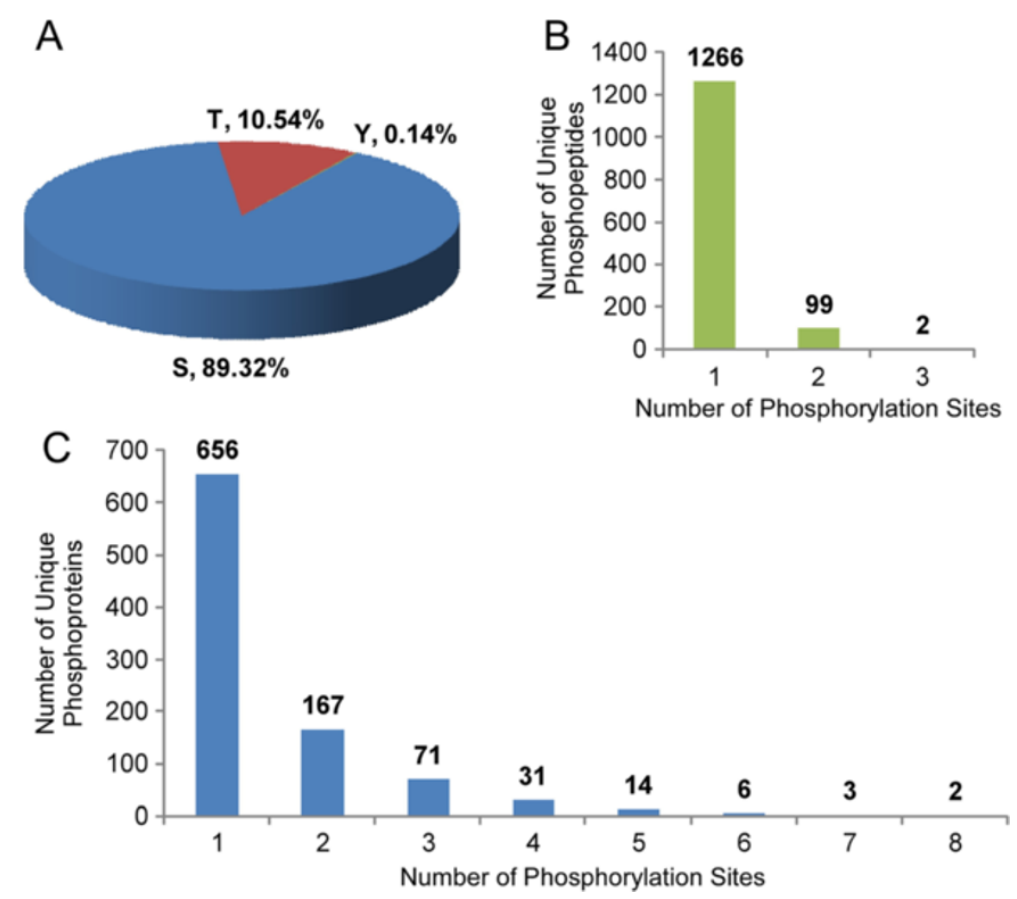

Figure 2 Analysis of identified phosphorylated sites. (A) Distribution of the phosphorylated amino acids. (B) Distribution of phosphorylation sites in the phosphopeptides. (C) Distribution of phosphorylation sites in the phosphoproteins.

identification. The review by Ghelis [39] also gave similar explanations for the lower phospho-Tyr proportion in plants. The distributions of phosphorylation sites in phosphopeptides and phosphoproteins were shown in Figure 2, panel B and C, respectively. Among the 1367 phosphopeptides, $1266(92.61 \%)$ of them each contained one phosphorylation sites, only 99 (7.24\%) contained two and $2(0.15 \%)$ contained three phosphorylation sites (Figure 2, panel B). From the phosphoprotein perspective, $656(69.05 \%)$ and $167(17.58 \%)$ of the 950 phosphoproteins were detected to have 1 and 2 phosphorylation sites, respectively. One hundred and twenty seven phosphoproteins contained more than two phosphorylation sites, of which 25 had at least five phosphorylation sites. Two phosphoproteins (Bradilg66870.1 and Bradi3g53907.1) each possessed 8 phosphorylation sites (Figure 2, panel C). The representative mass spectra of all the seven phosphopeptides of Bradilg66870.1 containing the 8 phosphorylated sites were shown in Additional file 2: Figure S1.

In order to obtain an overview of phosphorylation events in seedling leaves of B. distachyon, all of the 950 identified phosphoproteins were used to perform GO, Pfam annotation. Finally, 840 of them were annotated by Blast2GO and 804 of them possessed Pfam domains (Additional file 1: Table S1, sheet B). Significantly (FDR adjusted $\mathrm{p}<0.05$ ) enriched GO items of biological process, molecular function and cellular component are shown in Figure 3. Both the percent and p-value of each item were displayed to evaluate these GO items. From the biological process perspective, "nucleobase, nucleoside, nucleotide and nucleic acid metabolic process (23.05\%, GO:0006139, FDR:2.6E - 43)", "response to stimulus (19.37\%, GO:0 050896, FDR:1.5E - 106)", "Protein modification process (15.36\%, GO:0006464, FDR:3.8E - 17)", "cellular component organization (14.84\%, GO:0016043, FDR:2.3E - 98)", "transport (14.21\%, GO:0006810, FDR:4.5E - 23)", and "cellular amino acid and derivative metabolic process (11.26\%, GO:0006519, FDR:9.9E - 66)" were over-represen ted (Figure 3, panel A). From the cellular component perspective, "membrane (32.63\%, GO:0016020, FDR:8.7E 99)" and "nucleus (9.37\%, GO:0005634, FDR:1.5E - 8)" were significantly enriched (Figure 3, panel B). From the molecular function perspective, "protein binding (25.57\%, GO:0005515, FDR:6.1E - 86)", "nucleotide binding (24.42\%, GO:0000166, FDR:3.1E - 22)", "kinase activity (11.58\%, GO:0016301, FDR:1.3E - 8)" and "DNA binding (11.37\%, GO:0003677, FDR:3E - 12)" were the highly enriched molecular function items (Figure 3, panel C).

\section{Conservation and KEGG pathway analyses of phosphoproteins}

To investigate the conservation of phosphoproteins between $B$. distachyon and other plant species, the sequences of the $950 \mathrm{~B}$. distachyon phosphoproteins were used as queries to blast a phosphoprotein database which were constructed based on datasets from P3DB (Plant Protein Phosphorylation DataBase) [40], MORE (Medicago-Omics Repository) [13] and PhosPhAt 


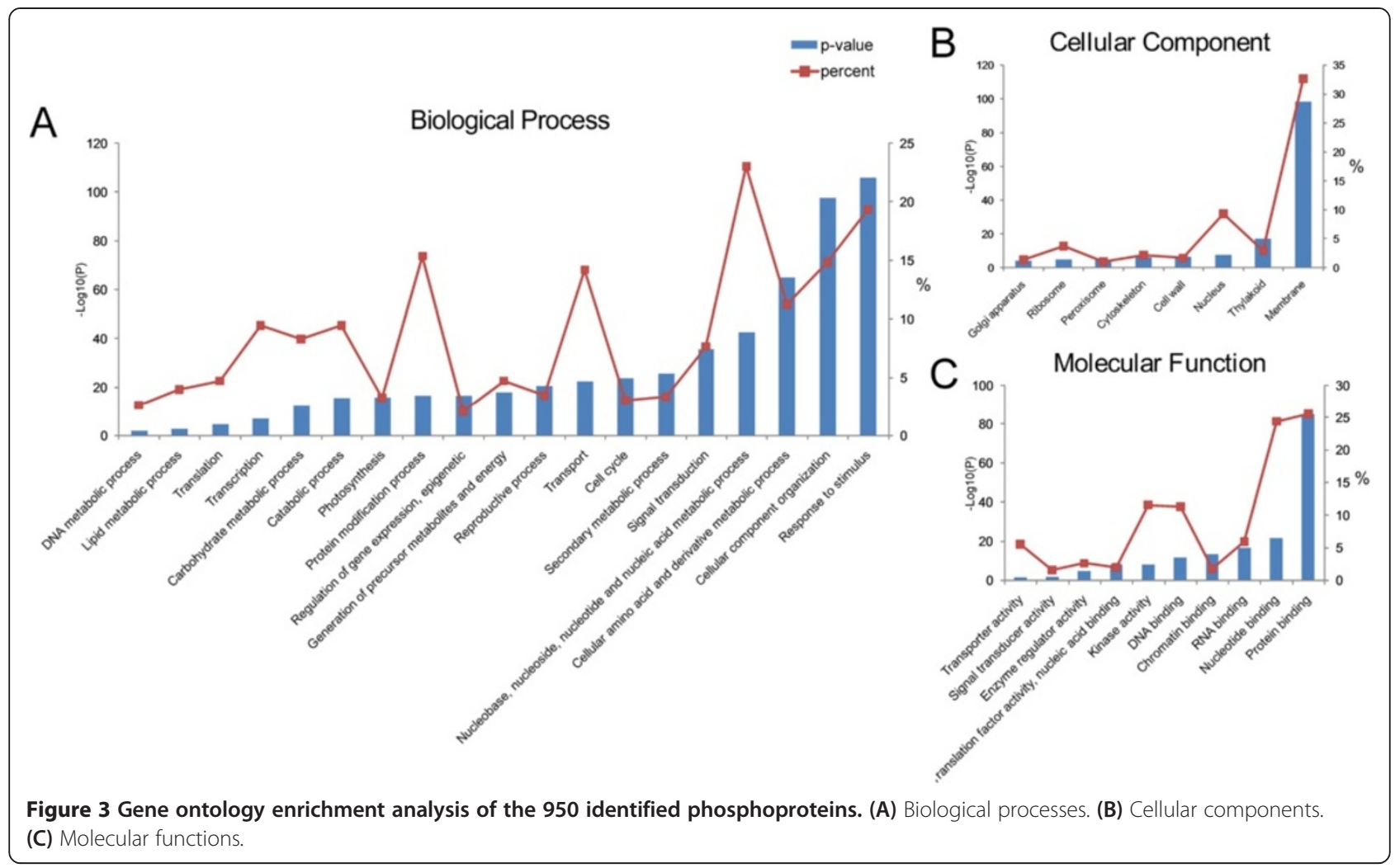

4.0 [41]. Oryza sativa and Arabidopsis thaliana, as the model species of monocots and dicots, respectively, possess more comprehensive phosphorylaton information than other species. Recently, a large phosphoprotein dataset of the model legume Medicago truncatula was released [13]. B. distachyon was therefore compared with the three species to determine the degree of conservation of the phosphoproteins among them. The threshold was set as Score $\geq 80$, E-value $<1 \mathrm{E}-10$ and Identity $\geq 30 \%$. Finally, 582 (61.3\%) phosphoproteins were identified with phosphorylation orthologs in all the three species, 202 (21.3\%) had phosphorylation orthologs in two of the three species, and $103(10.8 \%)$ had phosphorylation orthologs in only one species (Additional file 3: Table S2). Only 63 phosphoproteins had no phosphorylation orthologs in the three species. The conserved analysis results are shown in Additional file 3: Table S2. Further biological process enrichment analysis of the 582 highly conserved phosphoproteins (Additional file 4: Figure S2, panel A) showed that "cellular protein modification process (GO:000 6464, FDR:2.4E-2)" and "signal transduction (GO:0007165, FDR:5.0E-3)" were significantly over-represented from the total identified phosphoproteins (Additional file 4: Figure S2, panel B).

To reveal the phosphoprotein-associated pathways, the 950 phosphoproteins were mapped to KEGG; 31 B. distachyon KEGG pathways with not less than 3 hits were highlighted by the phosphoproteins identified in this study (Additional file 5: Table S3 and Additional file 6: Figure S3). Many fundamental biological pathways were highlighted by the mapping of phosphoproteins from this work, including carbohydrate metabolism involving carbon metabolism (Additional file 6: Figure S3, panel D), glycolysis/gluconeogenesis (panel G), pyruvate metabolism (panel $\mathrm{N}$ ) and pentose phosphate pathway (panel P), energy metabolism including carbon fixation (panel I), starch and sucrose metabolism (panel L) and oxidative phosphorylation (panel R), and nucleotide metabolism including purine metabolism (panel $\mathrm{M}$ ). For genetic information processing, transcription in spliceosomes (panel A), translation related to RNA transport (panel C), ribosome (panel E) and mRNA surveillance (panel F) were highly enriched. Two signal transduction pathways were particularly evident: the plant hormone signal transduction pathway (panel Q) and the phosphatidylinositol signaling system (panel AC). Further analysis of the 582 highly conserved phosphoproteinsrelated pathways showed that carbohydrate metabolism and energy metabolism-associated pathways, splicing, RNA transport and mRNA surveillance pathways stood out (Additional file 5: Table S3). The result of KEGG pathway analysis was consistent with that from GO analysis, indicating that the methods used here were effective and reliable. 


\section{Phosphorylation of transcription factors}

In growth and development of plants, many transcription factors (TFs) are affected through phosphorylation by PKs or dephosphorylation by protein phosphatases (PPs), which in turn either positively or negatively regulate TF activity in facilitating a program of gene expression that results in changed cell behavior [42]. Based on GO and KEGG pathway analyses, transcription-associated proteins were one of the most significant groups of all phosphoproteins, and TFs particularly were the main constituents. Fifty eight TFs belonging to 21 different families were identified (Table 1) based on alignments of our phosphoproteins with $B$. distachyon TFs in PlantTFDB (http://planttfdb.cbi.pku.edu.cn/index.php?sp=Bdi) [43]. In PlantTFDB, there are 1751 B. distachyon TFs classified into 56 families. To determine the TF families significantly regulated by phosphorylation, a TF family enrichment test was performed from $21 \mathrm{TF}$ families contained 58 TFs compared with the background set (1751 TFs from 56 TF families in $B$. distachyon). The number of phosphorylated TFs belonging to a TF family was compared against the total number of TFs in that family of B. distachyon. Finally, 6 significantly over-represented $(\mathrm{p}<0.05)$ TF families (C3H, Trihelix, CAMTA, TALE, MYB_related, and CPP) were found (Figure 4, panel A). Bd21 is diploid inbred line with its genome size about $272 \mathrm{Mb}$ and contains 26,552 gene loci, coding for 31,029 distinct mRNA molecules [18]. Genes of all the $58 \mathrm{TFs}$ were localized in the five Brachypodium chromosomes (Figure 4, panel B). The distribution of the six significantly over-represented TF families had no obvious chromosomal preference.

\section{Phosphorylation motif enrichment analysis}

To date, about 14,000 and 12,000 phosphorylation sites representing nearly 4100 and 4900 phosphoproteins in P3DB have been found in A. thaliana and $O$. sativa, respectively [40]. For A. thaliana, PhosPhAt 4.0 contains 12,613 experimental phosphorylation sites and 5663 phosphoproteins [41]. In addition, a large dataset for the model legume $M$. truncatula containing 13,506 phosphorylation sites localized in 3926 phosphoproteins was available in MORE [13]. Phosphorylation modification of these sites are regulated by PKs, which account for about 5.5\% of the Arabidopsis genome [44]. Different PKs have preference for specific substrates, and many kinaseassociated phosphorylation motifs have been deciphered [45-49]. The Motif-X online tool was used in this study to identify the phosphorylation motifs, and 13 different motifs were enriched, including 11 Ser and 2 Thr motifs (Figure 5 and Additional file 7: Table S4, sheet A). The phosphoproteins containing each of the 13 motifs (listed in Additional file 7: Table S4, sheet B) were further used in GO enrichment analysis. As shown in Additional file 8: Figure S4, DNA binding or RNA binding was enriched from phosphoproteins with the motifs $1,2,3,6,10$ or 13 , whereas translation factor activity was enriched from phosphoproteins with the motifs 9 or 10. Phosphoproteins containing motifs 1,4 or 9 were mainly involved in the cell cycle and located in ribosomes. Lipid binding and receptor activity were over-represented from motifs 2 and 8, respectively, and tropism was enriched from phosphoproteins containing the motif 12 . Organic cyclic compound binding and heterocyclic compound binding functions were enriched from the novel motif 5 . No GO terms were significantly enriched from phosphoproteins containing motifs 7 or 11.

According to the literature and databases [45-49], 10 of these motifs were specific to known PKs and 3 had no corresponding known kinases (Additional file 7: Table S4, sheet A). Ten known motifs included proline-directed motifs such as [s/tP] (Motifs 6 and 13) and [Pxs/tP] (Motifs 1 and 12), which were potential substrates of mitogenactivated PK (MAPK), cyclin-dependent kinase (CDK) and CDK-like kinase. Basic motifs [sPR] (Motif 2) and [sPxR] (Motif 4) were recognized by growth-associated histone kinase (GHK), CDK or cell division cycle 2 (CDC2) kinase. [Rxxs] (Motif 8) was recognized by calcium/calmodulindependent PK II (CaMK-II). [MxRxxs] (Motif 7) was recognized by C-terminal Src Kinase-homologous Kinase 1 (CHK1). Acidic motifs [sDxE] (Motif 9) and [sxD] (Motif 10) were recognized by casein kinase-II (CK-II). The other 3 phosphorylation motifs had no known specific kinases and were therefore regarded as novel phosphorylation motifs found in $B$. distachyon. Further analysis of these motifs showed that [RxxsP] (Motif 3), as a basic motif, is a combination of [sP] (Motif 6) and [Rxxs] (Motif 8); and this motif may be recognized by MAPK, CDK or CaMK. [GxxsP] (Motif 5) is similar to [GsP], which is recognized by glycogen synthase kinase 3 (GSK-3), MAPK or CDK5. [sxG] (Motif 11) may be recognized by CKII [50]. Further inspection of the 127 phosphoproteins containing multiple phosphorylation sites (Additional file 1: Table S1, sheet B) showed that 122 of them possessed at least one of the 13 phosphorylation motifs (Additional file 7: Table S4, sheet C). Among them, 91 phosphoproteins possessed two or more kinds of motifs. Phosphorylation sites of most phosphoproteins were motif-specific, but some other phosphoproteins each contained multiple phosphorylation sites belonging the same motif. Two phosphoproteins (Bradilg51560.1 and Bradi3g03690.1) each contained four phosphorylation sites specific to one motif (Additional file 7: Table S4, sheet $C$ ). This suggests that most phosphoproteins with multiple sites are regulated by multiple PKs, and a small part of them are activated by only one kind of PKs. The phosphoprotein Bradilg66870.1 contained eight phosphorylation sites belonging to five kinds of motifs (Figure 6, panel A), which may be regulated by different PKs such as 


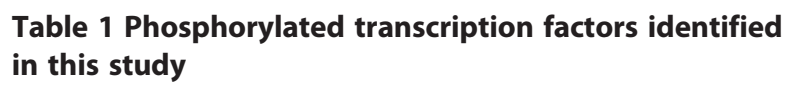

Table 1 Phosphorylated transcription factors identified in this study

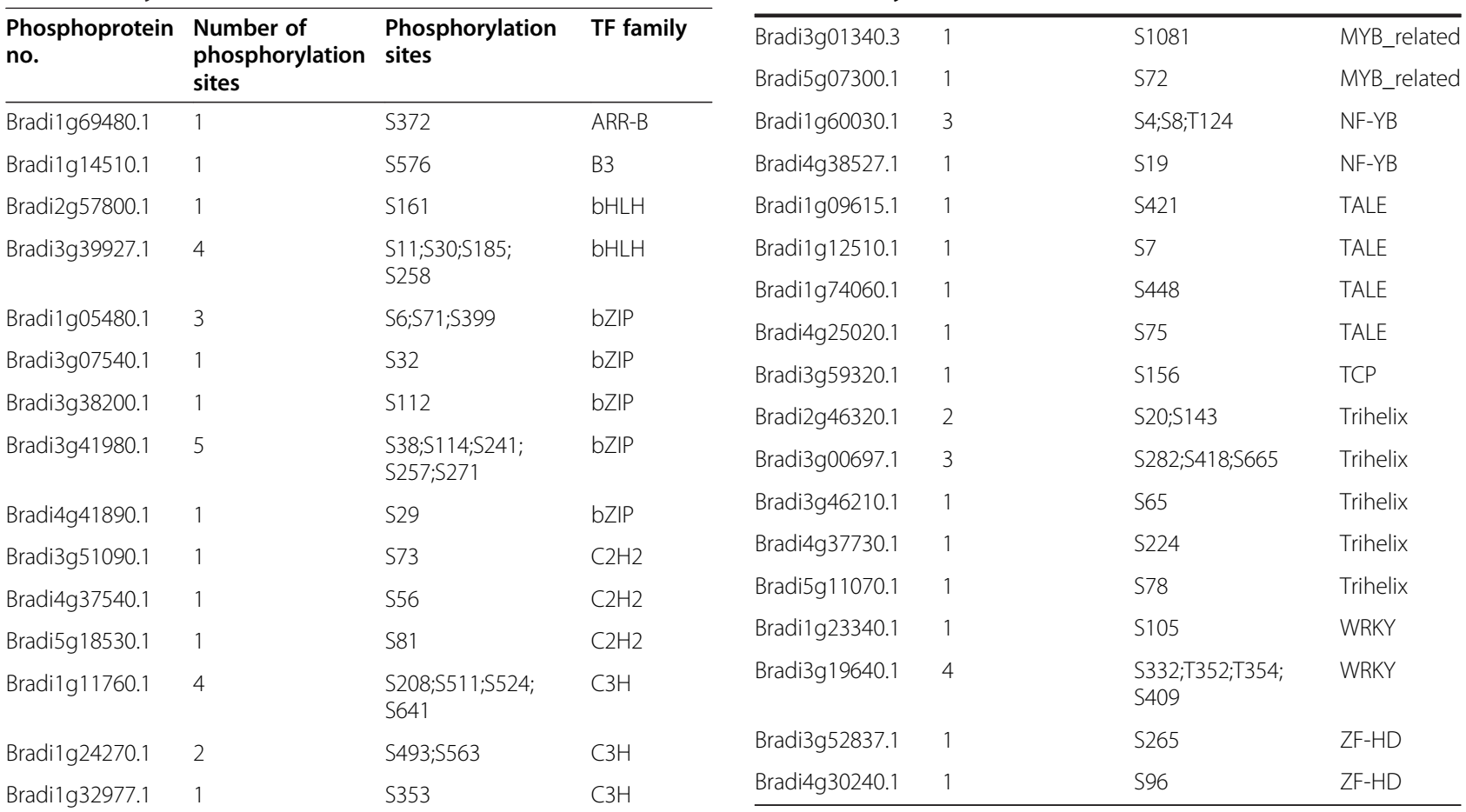

Table 1 Phosphorylated transcription factors identified in this study (Continued)

$\$ 435 \quad \mathrm{C} 3 \mathrm{H}$

S397; $5550 \quad \mathrm{C} 3 \mathrm{H}$

S206; $5286 \quad \mathrm{C} 3 \mathrm{H}$

$\mathrm{S} 258 \quad \mathrm{C} 3 \mathrm{H}$

$\mathrm{S} 38-\mathrm{C} 3 \mathrm{H}$

S437; $5448 \quad \mathrm{C} 3 \mathrm{H}$

S215;S219 C3H

T35 C3H

S19;:S21;S38;S42;S46 C3H

S329;5777;5929 CAMTA

S594:5973 CAMTA

$5956 \quad$ CAMTA

S234 CPP

S226 CPP

S190 G2-like

S100;\$358 GeBP

T86 GeBP

$5952 \quad$ HB-other

$5863 \quad$ HD-ZIP

S230 HSF

S416 MYB

S204 MYB_related

S265 MYB_related

S426 MYB_related

S105 MYB_related
MAPK, CDK and CK-II. Conservation analysis showed that phosphorylation orthologs in other species were also identified as phosphoproteins with many phosphosites, and 5 of the 8 identified phosphorylation sites in Bradilg66870.1 were also identified in other species whereas the other three were the newly identified phosphorylation sites present only in B. distachyon (Figure 6, panel B).

\section{Phosphorylation of PKs and PPs}

There are 989 PKs and 131 PPs in Arabidopsis according to PlantsP (http://plantsp.genomics.purdue.edu/) [51]. In this study, 84 PKs and 9 PPs were identified as phosphoproteins (Table 2). To identify the interactions between the phosphorylated PKs and PPs and their potential substrates, protein-protein interaction (PPI) analysis was conducted by using the Search Tool for Retrieval of Interacting Genes/Proteins (STRING). All 478 euKaryotic Orthologous Groups (KOGs) (Additional file 1: Table S1, sheet B) representing 685 of the 950 phosphoproteins were used to construct the phosphoprotein interaction network. To improve the accuracy of PPI analysis, the confidence level (score) was set to a high value $(0.900)$. Finally, a complicated PPI network centered by PKs and PPs was constructed and displayed by using Cytoscape software (Additional file 9: Figure S5). Depending on the homology comparison with the PKs and PPs of Arabidopsis in the PlantsP database, the phosphorylated PKs and PPs identified in our study were classified into six classes (Table 1): transmembrane receptor kinases and related non-transmembrane 


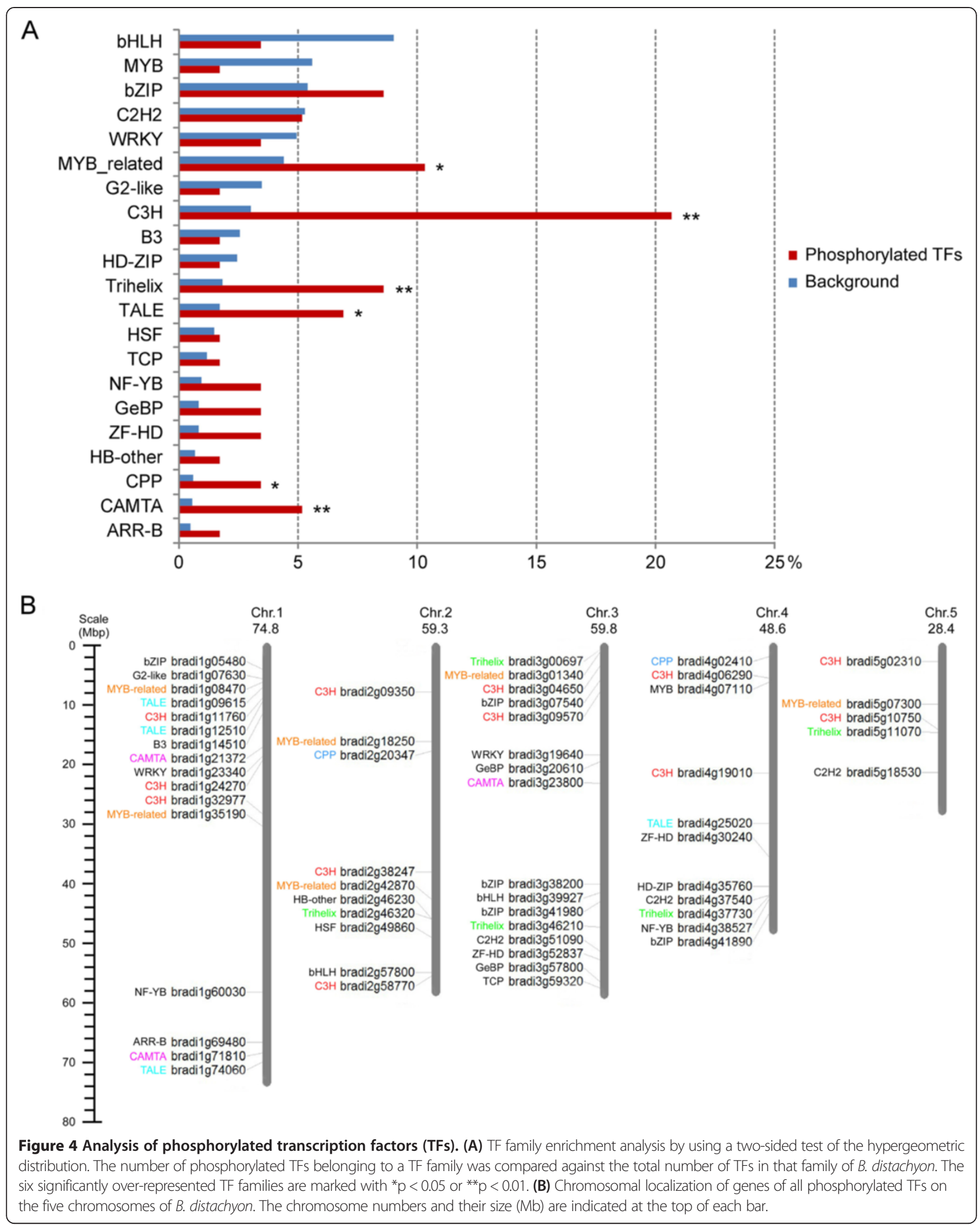




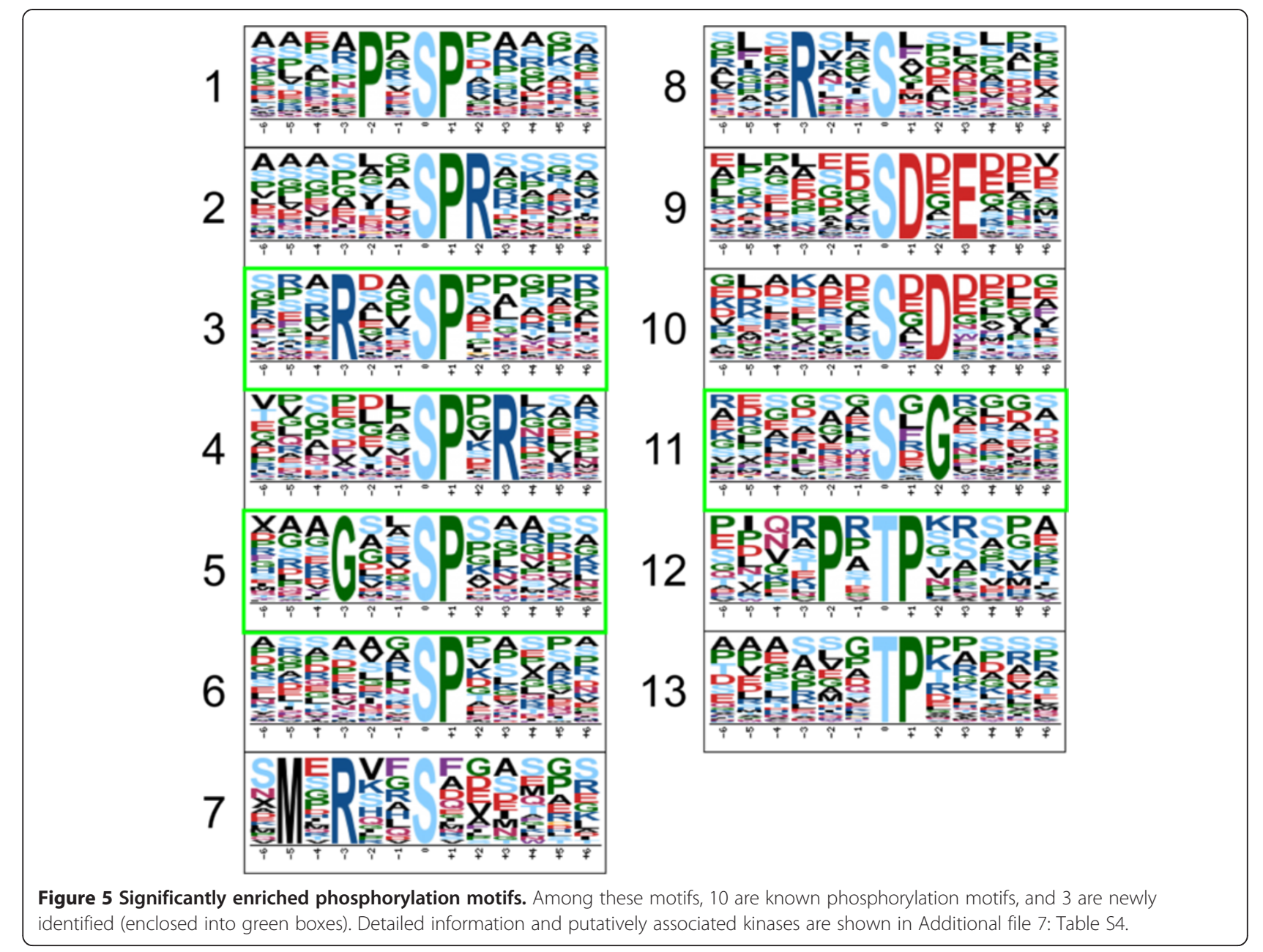

kinases, ATN1/CTR1/EDR1/GmPK6-like kinases, casein kinase I, non-transmembrane PKs, other kinases, and plant phosphatases.

MAPK cascades are universal signal transduction modules in plants and are involved in responses to various biotic and abiotic stresses, hormones, cell division and developmental processes [52]. Activation of a MAPK cascade can lead to changes in gene expression relating to cell wall biogenesis $[53,54]$. MAPK cascades contained three PK families, the MAPK, MAPK kinase (MAP2K) and MAPK kinase kinase (MEKK) families. In this study, one MAPK (Bradi1g49100.1; KOG0660), one MAP2K (Bradi1g51000.1; KOG0581) and five MEKK (Bradilg 10970.1, Bradi4g22760.1, Bradi5g10670.1, Bradi5g181 80.1, and Bradi5g24870.1; KOG0198) were identified as phosphoproteins, and conserved among $B$. distachyon, $O$. sativa, A. thaliana and M. truncatula (Additional file 3: Table S2), clearly indicating the fundamental roles of MAPK cascades in plants. A sub-network (Figure 7) centered by MEKK, MAP2K and MAPK was extracted from the whole PPI network mentioned above. From the sub-network, the MAPK family was the crucial PK in MAPK cascades and directly activated the substrates, so most phosphoproteins including many other kind of PKs (CDK, PHOT and STE20) interacted with MAPK. MAPK and many other PKs such as CK1 and SNT7 and PP2C were activated by MAP2K whereas MAP2K was activated by MEKK. Many phosphoproteins interacted simultaneously with MEKK and MAPK (Group 1) or MAP2K and MAPK (Group 2), and some interacted with MEKK, MAP2K and MAPK (Group 3). Several PKs and PPs, including GSK3, PDK1, DSPK and PP1, were also regulated by both MEKK and MAPK whereas only DSPP was regulated by both MAP2K and MAPK. Two phosphorylation sites (Table 2 and Additional file 1: Table S1, sheet A) were identified from BdMAPK (Bradilg49100.1) and one of them was phosphorylated at Tyr220. An Arabidopsis MAPK (AtMPK4) is phosphorylated at a Tyr residue during the activation process [55]. A previous study revealed that plant MAPKs contained a conserved phosphorylation motif, [tD/Ey] [56]. Interestingly, the BdMAPK we identified also contains this motif. Besides, we identified phosphorylation of 11 BdCDPKs and 4 BdCDKs, which may function in 


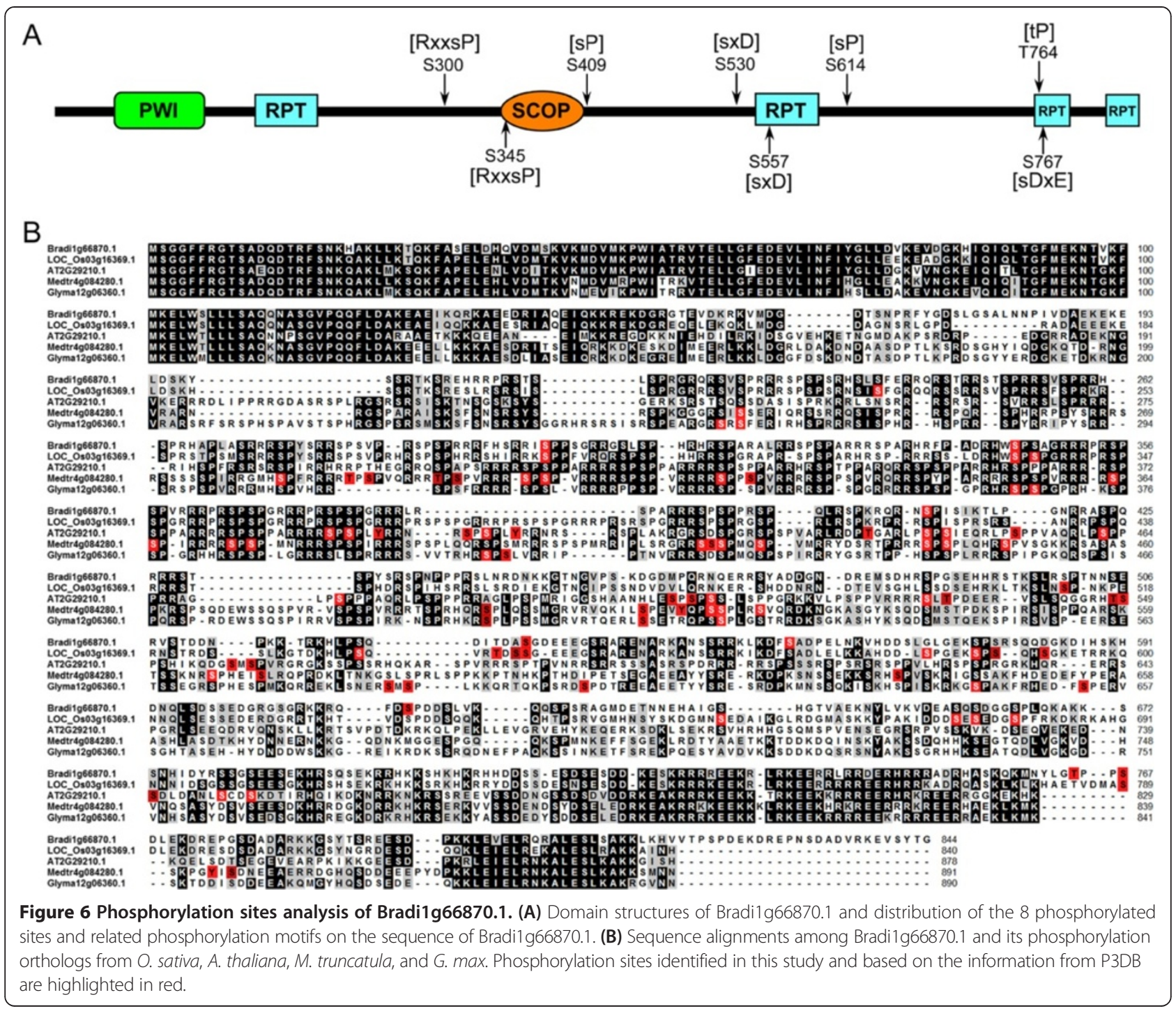

defense response and regulation of cell division and differentiation [57-59].

\section{Phosphorylation of signal transduction proteins}

In plants, many signal pathways are involved in vegetative growth. In the present study, KEGG pathway analysis showed that the plant hormone signal transduction pathway (Additional file 6: Figure S3, panel Q) and phosphatidylinositol signaling system (Additional file 6: Figure S3, panel AC) played important roles through phosphorylation modification of some crucial proteins. The hormones include cytokinin, abscisic acid, ethylene and brassinosteroid which mainly regulate cell division, cell elongation and shoot initiation. A homolog of type-B Arabidopsis response regulator (B-ARR, Bradilg69480.1) was found in the cytokinin regulated pathway. B-ARR is a DNA-binding transcriptional regulator, whose activity is regulated by phosphorylation of its phospho-accepting receiver domain through the His-kinase-mediated His to Asp phosphorelay and lack of this domain disturbs the development of young seedlings [60]. Likewise, other conserved phosphoproteins including a bZIP TF (Bradi3g38200.1), two ethylene-insensitive protein (Bradi4g 08380.1 and Bradi5g00700.1) and three serine/threonine PKs (Bradilg07620.1, Bradi3g32330.1 and Bradi2g56267.1) were involved in the abscisic acid, ethylene or brassinosteroid regulated signal transduction pathways. Another signal transduction pathway shown by KEGG was the phosphatidylinositol signaling system (Additional file 6: Figure S3, panel AC) that is associated with cell proliferation and differentiation [61]. Phosphatidylino sitol-3,4,5-trisphosphate 3-phosphatase (PTEN, Bradi 4g08080.1) acts as a lipid phosphatase to produce phosphatidylinositol 4,5-biphosphate (PtdIns(4,5)P2). Phos phatidylinositol-4- phosphate 5-kinase (Bradi1g44047.1) is involved in PtdIns(4,5)P2 synthesis. Phosphoinositide 
Table 2 Phosphorylated PKs and PPs identified in this study

\begin{tabular}{l}
\hline Phosphoprotein no. \\
\hline Class 1-transmembrane receptor kinases \\
Bradi1g07010.1 \\
Bradi1g20750.1 \\
Bradi1g35477.1 \\
Bradi1g58260.1 \\
Bradi1g59210.1 \\
Bradi1g60397.2 \\
Bradi1g72430.1 \\
Bradi1g76660.1 \\
Bradi2g01180.1 \\
Bradi2g12120.1 \\
Bradi2g27350.1 \\
Bradi2g34630.1 \\
Bradi2g43110.1 \\
Bradi2g47000.1 \\
Bradi3g08660.1 \\
Bradi3g32330.1 \\
Bradi3g40370.1 \\
Bradi3g44250.1 \\
Bradi3g49160.1 \\
Bradi3g53350.1 \\
Bradi4g35050.1 \\
Bradi5g03410.1 \\
Bradi5g12540.1 \\
Cradi5g13960.1
\end{tabular}

Phosphorylation site count

Phosphorylation sites

KOG no.

S372

$\mathrm{S} 735$

S697

$\mathrm{S741;S743;S733}$

S837

S345; $\$ 370$

S382;S389

$\mathrm{S} 770$

$\mathrm{S} 133$

$\$ 149$

S444

T263

S654;T674

S682

S1031

\$233

S93

S508

S693

S546:S535

S24

S342;T666;T672

S665;:S325

S612

S514;,S503

$\$ 12$

S1105;S761;S569

\$477

T14

S333;S410

S129

S478;S481;S400

S811

S27;\$153

S23;S37;S234;T417

S415

S417

S456

S10
KOG1187

KOG1187

KOG1187

KOG1187

KOG1187

KOG1187

KOG1187

KOG1187

KOG1187

KOG1187

KOG1187

KOG1187

KOG1187

KOG1187

KOG1187

KOG1187

KOG1187

KOG1187

KOG1187

KOG1187

KOG1187

KOG1187

KOG1187

KOG1187

KOG0192

KOG0192

KOG0192

KOG0192

KOG0192

KOG0192

KOG0192

KOG0192

KOG0192

KOG0192

KOG0192

KOG1163

KOG1163

KOG1163

KOG1164

KOG0032 
Table 2 Phosphorylated PKs and PPs identified in this study (Continued)

\begin{tabular}{|c|c|c|c|}
\hline Bradi1g24240.1 & 1 & S524 & KOG0032 \\
\hline Bradi1g35090.1 & 4 & S102;S26;T36;T46 & KOG0032 \\
\hline Bradi1g56970.1 & 2 & S557;S85 & KOG0032 \\
\hline Bradi2g15520.1 & 1 & S537 & KOG0032 \\
\hline Bradi4g24390.1 & 1 & S504 & KOG0032 \\
\hline Bradi4g39870.1 & 1 & S538 & KOG0032 \\
\hline Bradi4g40300.1 & 1 & S509 & KOG0032 \\
\hline Bradi5g19430.1 & 4 & S40;S549;S553;S16 & KOG0032 \\
\hline Bradi1g10970.1 & 1 & S230 & KOG0198 \\
\hline Bradi2g26547.1 & 1 & S972 & KOG0198 \\
\hline Bradi4g22760.1 & 1 & S155 & KOG0198 \\
\hline Bradi5g10670.1 & 1 & S607 & KOG0198 \\
\hline Bradi5g18180.1 & 2 & S335;\$337 & KOG0198 \\
\hline Bradi5g24870.1 & 1 & S893 & KOG0198 \\
\hline Bradi1g26670.1 & 1 & S322 & KOG0201 \\
\hline Bradi1g42257.2 & 3 & S502;S336;S477 & KOG0581 \\
\hline Bradi1g51000.1 & 1 & T25 & KOG0581 \\
\hline Bradi1g75150.1 & 1 & T30 & KOG0581 \\
\hline Bradi1g77700.1 & 3 & S538;S562;S625 & KOG0582 \\
\hline Bradi3g05890.1 & 2 & S330;T331 & KOG0582 \\
\hline Bradi3g31110.1 & 1 & S537 & KOG0582 \\
\hline Bradi1g07620.1 & 2 & S179;S183 & KOG0583 \\
\hline Bradi2g56267.1 & 1 & S158 & KOG0583 \\
\hline Bradi1g23970.1 & 1 & S614 & KOG0584 \\
\hline Bradi2g56460.1 & 1 & S394 & KOG0592 \\
\hline Bradi1g18450.2 & 1 & S375 & KOG0600 \\
\hline Bradi1g62440.1 & 1 & S77 & KOG0600 \\
\hline Bradi2g12937.2 & 1 & S509 & KOG0600 \\
\hline Bradi2g26680.1 & 1 & T198 & KOG0600 \\
\hline Bradi2g61310.1 & 2 & S93;T198 & KOG0600 \\
\hline Bradi1g11380.1 & 3 & S635;S654;T28 & KOG0606 \\
\hline Bradi4g45310.1 & 4 & S378;S385;S286;S297 & KOG0610 \\
\hline Bradi5g07360.1 & 3 & S508;S306;T304 & KOG0610 \\
\hline Bradi3g10350.1 & 2 & S466;S427 & KOG0614 \\
\hline Bradi2g38590.1 & 1 & Y235 & KOG0658 \\
\hline Bradi2g26510.1 & 2 & S337;T173 & KOG0659 \\
\hline Bradi1g49100.1 & 2 & T218;Y220 & KOG0660 \\
\hline Bradi5g13980.1 & 3 & $\mathrm{S125;S190;S693}$ & KOG0663 \\
\hline Bradi1g10530.1 & 1 & S546 & KOG0667 \\
\hline Bradi4g00230.1 & 1 & T544 & KOG0670 \\
\hline Bradi2g42320.1 & 1 & S33 & KOG0671 \\
\hline \multicolumn{4}{|c|}{ Class 5-other kinases } \\
\hline Bradi1g08540.1 & 1 & S92 & KOG1151 \\
\hline Bradi1g26580.1 & 1 & S30 & KOG1166 \\
\hline Bradi2g17660.1 & 3 & S545;T531;T540 & KOG0594 \\
\hline
\end{tabular}


Table 2 Phosphorylated PKs and PPs identified in this study (Continued)

\begin{tabular}{|c|c|c|c|}
\hline \multicolumn{4}{|c|}{ Class 6-plant phosphatases } \\
\hline Bradi2g36370.1 & 2 & S847;S500 & KOG0374 \\
\hline Bradi1g24400.1 & 1 & S414 & KOG0698 \\
\hline Bradi1g47710.1 & 1 & S339 & KOG0698 \\
\hline Bradi3g39540.1 & 1 & S504 & KOG0698 \\
\hline Bradi3g52110.1 & 2 & $\mathrm{~S} 121 ; \mathrm{S} 131$ & KOG0700 \\
\hline Bradi1g42810.1 & 1 & S232 & KOG1716 \\
\hline Bradi2g37450.1 & 3 & S283;S465;T92 & KOG1716 \\
\hline Bradi4g08080.1 & 2 & S73;S113 & KOG2283 \\
\hline
\end{tabular}

phospholipase C (PLC, Bradi1g16560.1) mediates the production of second messenger molecules diacylglycerol (DAG) and inositol 1,4,5-trisphosphate (IP3) by hydrolysis of PtdIns(4,5)P2. The three enzymes were all identified as phosphoproteins (Additional file 1: Table S1).

\section{Phosphorylation of proteins related to cell wall expansion} The Poaceae family can be distinguished from most other land plants by the composition of their cell walls [62,63]. B. distachyon is a good model for cell wall (mostly primary wall) studies of temperate grasses [24,64]. Cell wall loosening is essential for leaf expansion during vegetative growth of plants, and this must be balanced with polymer synthesis and wall restrengthening to prevent the cell wall from rupture. Multiple receptor-like PKs (RLKs) are implicated in cell wall signaling [30]. The RLK (Bradi2g12120.1), a conserved phosphoprotein among $B$. distachyon, $O$. sativa and $A$. thaliana, was phosphorylated at Ser149 (Table 2). Hématy et al. [65] found a RLK (THE1) mediating the response of growing plant cells to inhibition of cellulose synthesis in Arabidopsis and that it can be phosphorylated at a Thr residue. The phosphorylation of RLK identified in our study may be involved in cell wall remodeling during leaf development. A wall-associated receptor kinase (WAK, Bradi3g49160.1), as a kind of RLK, has the potential to serve as both linkers of the cell wall to the plasma membrane and as a signaling molecule. WAKs also function in pathogen response and cell expansion and elongation $[27,66,67]$. WAK proteins contain a cytoplasmic Ser/Thr kinase (STK) domain, a transmembrane domain, and an extra cytoplasmic region with several epidermal growth factor (EGF) repeats [28,68]. Interestingly, the

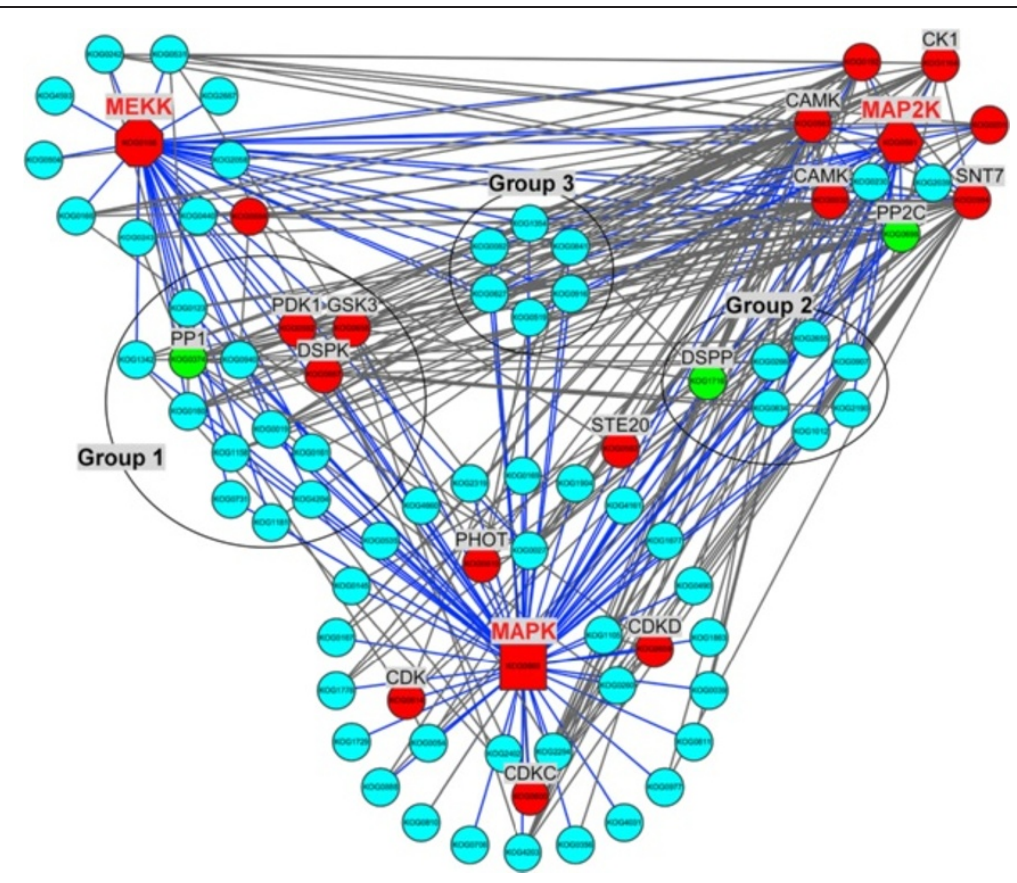

Figure 7 Phosphorylation regulatory network centered by MAPK cascade. Protein kinases and protein phosphatases are highlighted in red and green, respectively and other phosphoproteins identified in this study are shown with sky-blue nodes. Edges with blue color represent the phosphoproteins with direct relationship to MEKK, MAP2K or MAPK. 
phosphorylated site of this BdWAK was located on the Cterminal (Ser693) in our study, which was outside these domains above. As a kind of RLK, phosphorylation of this BdWAK may regulate functions of itself and further mediate the phosphorylation of enzymes involved in synthesis of cellulose in cell walls.

The primary cell walls of grasses consist mainly of cellulose, arabinoxylan and uniquely $(1,3 ; 1,4)-\beta$-D-glucan, small quantities of structural proteins, and low levels of pectin and xyloglucan $[63,69-71]$. Cellulose is by far the most abundant biological polymer on earth, and is synthesized by cellulose synthase (CESA) contained in plasma membrane-localized complexes. In A. thaliana, CESA1, CESA3 and CESA6 interact with each other and are largely responsible for cellulose production during formation of the primary cell wall [72-75], whereas three types of CESA subunits (CESA4, CESA7 and CESA8) are required for secondary cell wall formation [74,76,77]. In addition, CESA2, CESA5, and CESA9 also function in primary cell wall synthesis [72]. This study identified 6 phosphoproteins as different CESAs in B. distachyon, namely BdCESA1, BdCESA2, BdCESA4, BdCESA7, Bd CESA8 and BdCESA9 (Bradi2g34240.1, Bradilg04597.1, Bradi2g49912.1, Bradi3g28350.1, Bradilg54250.1 and Bradi 4g30540.1 in Additional file 1: Table S1). Representative MS/MS spectra of phosphopeptides BdCESA2 and BdCE SA4 are shown in Additional file 10: Figure S6, panel A and $\mathrm{B}$, respectively. AtCESA2 expressed in all organs except root hairs, and lack of AtCESA2 expression can lead to stunted growth of hypocotyls in seedlings and greatly reduced seed production in mature plants [78]. All cellulose synthases described to date have shown conserved structural features [78]. The amino N-terminal of the CESA protein contains a RING finger domain with a strongly conserved $\mathrm{CxxC}$ motif $(\mathrm{C}=\mathrm{Cys}, \mathrm{x}=$ any amino acid) [79]. The RING finger domain is a small zincbinding domain found in many functionally distinct proteins [80]. Kurek et al. [81] confirmed that GhCESA1 formed homodimers as well as heterodimers with GhCESA2 through a zinc-binding domain. Chu et al. [78] demonstrated that AtCESA2 could homodimerize and the zinc finger domain of AtCESA2 was important for the interaction. Sequence analyses indicated that all six identified BdCESAs contained a RING finger domain with a conserved CxxC motif (Additional file 10: Figure S6, panel C). There are 8 transmemberane regions in BdCESA1, BdCESA2, BdCESA8 and BdCESA9, but there is no the second transmemberane region in BdCESA4 and BdCE SA7. All phosphorylation sites identified from these BdCESAs are located between the RING finger domain and transmemberane region I. Nühse et al. [8] performed a plasma membrane phosphoproteomics in Arabidopsis and revealed that CESA1, CESA3, and CESA5 proteins were phosphorylated at several sites clustered in the two hypervariable regions. Chen et al. [82] found that phosphorylation of CESA1 differentially affects polar interaction with microtubules and may regulate the length or quantity of a subset of cellulose microfibrils in the primary cell wall. The region with phosphorylation sites in each BdCESA belonged to hypervariable region I (Additional file 10: Figure S6, panel C). Phosphorylation of AtCESA7 was linked to its degradation via a $26 \mathrm{~S}$ proteasomedependent pathway [83]. It seems that phosphorylation of the 6 BdCESAs may play vital roles in cell expansion during rapid vegetative growth of Brachypodium.

$(1,3 ; 1,4)-\beta$-D-glucan is mainly detected in some families of the Poales [70]. Recently, various genes involved in $(1,3 ; 1,4)-\beta-D-$ glucan synthesis were identified $[84,85]$. They belong to two grass-specific families (CSLF and CSLH) of the cellulose synthase-like (CSL) gene superfamily. In the present study, two CSL proteins BdCSLF6 (Bradi3g16307.1) and BdCSLH1 (Bradi5g10130.1) were identified as phosphoproteins. In developing barley coleoptiles, the transcript levels of $H \nu C s l F 6$ are maximal in 4- to 5 - $\mathrm{d}$ coleoptiles, at the time when $(1,3 ; 1,4)-\beta$ $\mathrm{D}$-glucan content of coleoptile cell walls also reaches its maximum level [84]. HvCSLH1 protein is responsible for $\beta$-glucan deposited in cell walls of transgenic Arabidopsis [85]. In B. distachyon, the CSLH gene showed a much higher transcript level and played a prominent role in $(1,3 ; 1,4)-\beta$-D-glucan synthesis [64].

\section{Conclusions}

In this work, $\mathrm{TiO}_{2}$-enriched phosphopeptides in $\mathrm{Bd} 21$ seedling leaves detected by LC-MS/MS, and MaxQuant software were used to identify the phosphoproteins and phosphorylation sites. To our knowledge, this is the first large-scale phosphoproteome analysis in B. distachyon and provides an overview of in vivo phosphorylation events in seedling leaves. A total of 950 phosphoproteins containing 1470 unambiguous phosphorylation sites were identified. In-depth GO, conservation, and KEGG pathway analyses of these phosphoproteins revealed the phosphorylation profiling during rapid seedling leaf growth. GO analysis showed that the 950 phosphoproteins mainly functioned in protein binding, nucleotide binding and kinase activity. Conservation analysis revealed that 887 (93.4\%) of the phosphoproteins had phosphorylation orthologs in other species. Many fundamental biological pathways and two signal transduction pathways were identified through KEGG pathway analysis.

Fifty eight TFs, 94 PKs and 8 PPs were identified from the large dataset and analyzed in depth. Thirteen enriched motifs specific for various Ser/Thr PKs were identified from $B$. distachyon. A total of 127 phosphoproteins contained multiple phosphorylation sites and 91 of them contained two or more phosphorylation motifs. Several signal transduction and cell wall expansion associated 
phosphoproteins that may have vital functions in the rapid growth of $B$. distachyon seedling leaves were found such as B-ARR, PTEN, CESA and CSL. Our overall results provide an important resource for further research and new insights into the regulation of protein post-translational phosphorylation during rapid vegetative growth of Poaceae species.

\section{Methods}

Plant materials

Seeds of Brachypodium distachyon Bd21, kindly provided by Dr. John Vogel, USDA-ARS, Albany, CA, were surface sterilized in $5 \%$ sodium hypochlorite for $5 \mathrm{~min}$, and rinsed 4 times in sterile distilled water. Seeds were submerged in water for $12 \mathrm{~h}$ at room temperature, and then transferred to wet filter paper for $24 \mathrm{~h}$ to germinate at room temperature $\left(22-25^{\circ} \mathrm{C}\right)$. Uniformly germinated seeds were selected and grown in three plastic pots containing Hoagland's solution [86]; the Hoagland's solution was changed every two days. At the three leaf stage, all three leaves were collected and frozen at $-80^{\circ} \mathrm{C}$. Culture of the $\mathrm{Bd} 21$ seedlings was repeated three times and the leaves were collected independently.

\section{Protein extraction}

Total proteins were extracted from seedling leafs according to the method of Wang et al. [87] with minor modifications. Approximately $400 \mathrm{mg}$ fresh leaves of each sample were ground into fine powder in liquid nitrogen. The ground powder was suspended in $4 \mathrm{~mL}$ SDS buffer (30\% sucrose, 2\% SDS, $100 \mathrm{mM}$ Tris- $\mathrm{HCl}, \mathrm{pH}$ 8.0, $50 \mathrm{mM}$ EDTA- $\mathrm{Na}_{2}, 20 \mathrm{mM}$ DTT) and $4 \mathrm{~mL}$ phenol (Tris-buffered, $\mathrm{pH} 8.0$ ) in a $10 \mathrm{~mL}$ tube, and $1 \mathrm{mM}$ phenylmethanesulfonyl fluoride (PMSF) and PhosSTOP Phosphatase Inhibitor Cocktail (Roche, Basel, Switzerland), were added to inhibit the activities of proteases and phosphatases. The mixtures were thoroughly vortexed thoroughly for $30 \mathrm{~s}$ and the phenol phase was separated by centrifugation at $14,000 \times g$ and at $4^{\circ} \mathrm{C}$ for $15 \mathrm{~min}$. The upper phenol phase was pipetted to fresh $10 \mathrm{~mL}$ tubes and four volumes of cold methanol plus $100 \mathrm{mM}$ ammonium acetate were added, and the mixture was stored at $-20^{\circ} \mathrm{C}$ for at least $30 \mathrm{~min}$. After centrifugation at $14,000 \times g$ and at $4^{\circ} \mathrm{C}$ for $15 \mathrm{~min}$, the supernatant was carefully discarded and the precipitated proteins were washed twice with cold methanolic ammonium acetate $(100 \mathrm{mM})$ and ice-cold $80 \%$ acetone, respectively. Finally the pellet was vacuum-dried and then dissolved in lysis buffer (7 M urea, $2 \mathrm{M}$ thiourea, $4 \% \mathrm{w} / \mathrm{v}$ CHAPS and $65 \mathrm{mM} \mathrm{DTT}$ ) over $3 \mathrm{~h}$ at $4^{\circ} \mathrm{C}$. The protein mixtures were harvested by centrifugation at $14,000 \times g$ and $4^{\circ} \mathrm{C}$ for $15 \mathrm{~min}$ to remove insoluble materials. The concentrations of the extracted protein mixtures were determined with a 2-D Quant Kit (Amersham Bioscience, Buckinghamshire, UK) using BSA (2 mg/mL) as standard, and the final protein solution was stored at $80^{\circ} \mathrm{C}$ for later use.

\section{Phosphopeptide enrichment using $\mathrm{TiO}_{2}$ microcolumns}

Extracted protein mixtures were directly reduced with dithiothreitol (DTT), alkylated with iodoacetamide, and subsequently digested with endoproteinase Lys-C and trypsin as previously described [88]. The enrichment procedure for phosphopeptides was performed as reported by Wu et al. [5] with modifications. The $\mathrm{TiO}_{2}$ beads (GL Sciences, Tokyo, Japan) were incubated in $400 \mu \mathrm{L}$ loading buffer containing $65 \%$ Acetonitrile $(\mathrm{ACN}) / 2 \%$ trifluoroaceticacid (TFA)/saturated by glutamic acid. A total of $2 \mathrm{mg}$ of tryptic peptides were dissolved in $600 \mu \mathrm{L}$ loading buffer, and incubated with an appropriate amount of $\mathrm{TiO}_{2}$ beads. After washing with $600 \mu \mathrm{L}$ wash buffer (65\% ACN/0.1\% TFA), phosphopeptides were eluted twice with $300 \mu \mathrm{L}$ elution buffer $\left(500 \mathrm{mM} \mathrm{NH}_{4} \mathrm{OH} / 60 \% \mathrm{ACN}\right)$ and the eluates were dried down and reconstituted in $0.1 \%$ formic acid (FA) $/ \mathrm{H}_{2} \mathrm{O}$ for MS analysis.

\section{Phosphopeptide identification and phosphorylation site localization using LC-MS/MS}

Enriched phosphopeptides were separated on a selfpacked C18 reverse phase column (75 $\mu \mathrm{m}$ I.D., $150 \mathrm{~mm}$ length) (Column Technology Inc., Fremont, CA), which directly connected the nano electrospray ion source to a LTQ-Orbitrap XL mass spectrometer (Thermo Fisher Scientific, San Jose, CA). Pump flow was split to achieve a flow rate at $1 \mu \mathrm{L} / \mathrm{min}$ for sample loading and $300 \mathrm{~nL} /$ min for MS analysis. The mobile phases consisted of $0.1 \%$ FA (A), and $0.1 \%$ FA and $80 \%$ ACN (B). A five-step linear gradient of $5 \%$ to $30 \% \mathrm{~B}$ in $105 \mathrm{~min}, 35 \%$ to $90 \% \mathrm{~B}$ in $16 \mathrm{~min}, 90 \% \mathrm{~B}$ in $4 \mathrm{~min}, 90 \%$ to $2 \% \mathrm{~B}$ for $0.5 \mathrm{~min}$ and $2 \%$ B for 14.5 min was performed. The spray voltage was set to $2.0 \mathrm{kV}$ and the temperature of the heated capillary was $240^{\circ} \mathrm{C}$.

For data acquisition, each MS scan was acquired at a resolution of 60,000 (at $400 \mathrm{~m} / \mathrm{z}$ ) with lock mass option enabled and was followed by a data-dependent top 10 MS/MS scans using collision induced dissociation (CID). The threshold for precursor ion selection was 500 and mass window for precursor ion selection was 2.0 Da. The dynamic exclusion duration was $120 \mathrm{~s}$, repeat count was 1 and repeat duration was $30 \mathrm{~s}$. The analyzer for the MS scans was Orbitrap and for the MS/MS scans LTQ (37\% relative collision energy). Three biological replicates were performed independently from sample collection to phosphopeptide identification using LC-MS/MS.

The raw files were processed with MaxQuant (version 1.2.2.5) [89] and searched against the $B$. distachyon protein database (31,029 entries in total) in Phytozome (http:// www.phytozome.net/search.php; version 9.1) concatenated with a decoy of reversed sequences. The following 
parameters were used for database searches: cysteine carbamidomethylation was selected as a fixed modification; methionine oxidation, protein $\mathrm{N}$-terminal acetylation, and phosphorylation on serine, threonine and tyrosine were selected as variable modifications. Up to two missing cleavage points were allowed. The precursor ion mass tolerances were $7 \mathrm{ppm}$, and fragment ion mass tolerance was $0.5 \mathrm{Da}$ for MS/MS spectra. The false discovery rate (FDR) was set to $<1.0 \%$ for both peptide and protein identifications, the minimum peptide length was set to 6 .

Phosphorylation site localization was based on PTM scores that assign probabilities for each of the possible sites according to their site-determining ions. In this study, MaxQuant (version 1.2.2.5) was used to calculate PTM scores and PTM localization probabilities. Potential phosphorylation sites were then grouped into three categories depending on their PTM localization probabilities $[10,37,88]$, namely class I (localization probability, $\mathrm{P} \geq 0.75)$, class II $(0.75>\mathrm{P} \geq 0.5)$ and class III $(\mathrm{P}<0.5)$. A false discovery rate (FDR) of $1 \%$ was used for phosphorylation sites identification.

\section{Bioinformatics}

The GO biological processes, molecular functions and cellular components of the identified phosphoproteins were examined by using Blast2GO software [90]. GO enrichment analysis was conducted with agriGO [91]. The statistical test method was set as Fisher and the multi-test adjustment method was set as Bonferroni. Pfam domain information was extracted from the database (http://pfam.sanger.ac.uk/search) [92]. Protein descriptions were extracted from the $B$. distachyon protein database (31,029 entries in total) in Phytozome (http:// www.phytozome.net/search.php; version 9.1). For pathway analysis, the proteins were searched in the KEGG $B$. distachyon database and mapped to B. distachyonspecific pathways with KEGG Mapper. The enrichment of TF family significantly regulated by phosphorylation was performed using a two-sided test of the hypergeometric distribution. The significantly enriched phosphorylation motifs were extracted from phosphopeptides with unambiguous phosphorylation sites (class I) using the Motif-X algorithm [33]. The phosphopeptides were centered at phosphorylated amino acid residues and aligned, and six positions upstream and downstream of the phosphorylation site were included. In the cases of $\mathrm{C}$ - and $\mathrm{N}$ terminal peptides, the sequence was completed to 13 amino acids with the required number of " $\mathrm{X}$ "s, where $\mathrm{X}$ represents any amino acid. Because the upload restriction of Motif-X is $10 \mathrm{MB}$, a fasta format dataset (nearly $10 \mathrm{MB}$ ) containing the protein sequences from the $B$. distachyon protein database in Phytozome (version 9.1) was used as the background database to normalize scores against random distributions of amino acids. The occurrence threshold was set to $5 \%$ of the input data set at a minimum of 20 peptides, and the probability threshold was set to $\mathrm{P}<10^{-6}$. The sequences of all the phosphoproteins were used for BLAST analysis with the $\mathrm{Na}$ tional Center for Biotechnology Information (NCBI) clusters of the KOG database to obtain the KOG numbers of those proteins. Then a dataset containing all the KOG numbers was used for protein-protein interaction (PPI) analysis by using the STRING database (version 9.1, http://string-db.org) [93]. Only interactions that had a confidence score of at least 0.9 were used to construct the network and the networks were then displayed using Cytoscape (version 3.0.2) software [94]. HMMTOP (version 2.0, http://www.enzim.hu/hmmtop/) was used to predict the transmembrane helices in proteins [95].

\section{Additional files}

Additional file 1: Table S1. Phosphorylation sites and phosphoproteins identified from seedling leaves of B. distachyon. (A) Total phosphorylation sites and phosphoproteins. (B) Annotation of the phosphoproteins.

Additional file 2: Figure S1. Representative tandem mass spectrometry spectra of the seven phosphopeptides containing the eight phosphosites in Bradi1g66870.1. (A) KHLPSQDITDAS(ph)GDEEEGSR. (B) HWS(ph)PSAGRR. (C) LKDFS(ph)ADPELNK. (D) MNYLGT(ph)PPS(ph)DLEK. (E) NS(ph)PISIK. (F) QFDS(ph)PDDSLVK. (G) RIS(ph)PPSGR.

Additional file 3: Table S2. Conservation analysis of phosphoproteins identified from $B$. distachyon.

Additional file 4: Figure S2. GO biological process enrichment of 582 highly conserved phosphoproteins. The statistical significance of the enrichment analysis is represented by a scale of red tones whose intensity is proportional to the degree of significance starting from $\mathrm{FDR}<0.05$.

Additional file 5: Table S3. Pathway analysis of the identified phosphoproteins by KEGG. (A) Pathway search results of all identified phosphoproteins and highly conserved phosphoproteins. (B) KEGG identifiers of all the identified phosphoproteins.

Additional file 6: Figure S3. Pathway mapping of identified phosphoproteins by KEGG. Phosphoproteins were analyzed in the KEGG $B$. distachyon pathway database. The phosphoproteins were used to search the KEGG B. distachyon database and mapped to $B$. distachyon specific pathways with KEGG Mapper. In each pathway map, objects with red foreground color and blue background color represent the highly conserved phosphoproteins; objects with pink foreground and blue background represent the other conserved phosphoproteins; objects with yellow foreground and blue background represent proteins identified as novel phosphoproteins; objects with green background represent the total proteins in the $B$. distachyon KEGG database. Details of the pathways can be found in Additional file 5: Table S3.

Additional file 7: Table S4. Enriched phosphorylation motifs, and the phosphopeptides and the corresponding phosphoproteins in each phosphorylation motif. (A) Phosphorylation motifs enriched by Motif- $X$ and the putative protein kinases. (B) Phosphopeptides and the corresponding phosphoproteins in each phosphorylation motif. (C) Motifs of phosphoproteins with multiple phosphorylation sites.

Additional file 8: Figure S4. GO enrichment of phosphoproteins represented by the phosphorylation motifs in $B$. distachyon. GO enrichment graphs of phosphoproteins contained each of 11 motifs were displayed by Blast2GO software. No GO terms were significantly enriched from phosphoproteins containing motif 7 or motif 11 . The statistical significance of the enrichment analysis is represented by a scale of red tones whose intensity is proportional to the degree of significance starting from $p<0.05$. 
Additional file 9: Figure S5. Protein-protein interaction network of the phosphoproteins. The confidence (score) was set as the highest (0.900) and only the phosphoproteins identified in this study were used to construct the interaction profiles. Protein kinases and protein phosphatases are highlighted in red and green respectively and other phosphoproteins identified in this study are shown with sky-blue nodes.

Additional file 10: Figure S6. Phosphorylation sites of the six BdCESAs. (A) and (B) The tandem mass spectrometry spectra of the phosphopeptides EFSGS(ph)LGNVAWK from BdCESA2 (Bradi1 g04597.1) and VTIASQLS(ph)DR from BdCESA4 (Bradi2g49912.1). (C) Sequence alignment among the six BdCESAs. Phosphorylation sites identified in this study are highlighted in red. The RING domain and transmembrane regions are highlighted in yellow and green, respectively.

\section{Abbreviations}

LC-MS/MS: Liquid chromatography-tandem mass spectrometry; GO: Gene ontology; KEGG: Kyoto Encyclopedia of Genes and Genomes; PK: Protein kinase; PP: Protein phosphatase; TF: Transcription factor; MAPK: Mitogenactivated protein kinase; CDK: Cyclin-dependent kinase; GHK: Growthassociated histone kinase; CDC2: Cell division cycle 2; CaMK: Calcium/ calmodulin-dependent protein kinase; CHK1: C-terminal Src Kinasehomologous Kinase 1; CK: Casein kinase; PPI: Protein-protein interaction; STRING: Search Tool for Retrieval of Interacting Genes/Proteins; KOG: EuKaryotic Orthologous Group; MAP2K: Mitogen-activated protein kinase kinase; MEKK: Mitogen-activated protein kinase kinase kinase; RLK: Receptor-like protein kinase; WAK: Wall-associated receptor kinase; CESA: Cellulose synthase; CSL: Cellulose synthase-like.

\section{Competing interests}

This manuscript has no financial or non-financial competing interests.

\section{Authors' contributions}

LDW and LX carried out all experiments, data analysis and wrote the manuscript. ZM and GAQ performed the protein extraction, $G O$ annotation and KEGG pathway analyses. ZSM and WC performed the conservation analysis. LXH helped English writing and proof reading of the manuscript. LDW and YYM conceived the study, participated in the design and coordination, and in interpretation of the dataset. All authors read and approved the final manuscript.

\section{Acknowledgements}

We wish to thank Dr. John Vogel, USDA-ARS, for providing the seeds of the inbred diploid Brachypodium distachyon line Bd21. We are grateful to Professor Robert Mclntosh, University of Sydney, Australia, for constructive suggestions on the manuscript. This research was financially supported by grants from the National Natural Science Foundation of China (31271703), the China-Australia Cooperation Project from Chinese Ministry of Science and Technology (2013DFG30530), and the National Key Project for Transgenic Crops of China (2011ZX08009-003-004). The data deposition to the ProteomeXchange Consortium was supported by PRIDE Team, EBI.

Received: 5 January 2014 Accepted: 6 May 2014

Published: 16 May 2014

\section{References}

1. Thingholm TE, Jensen ON, Larsen MR: Analytical strategies for phosphoproteomics. Proteomics 2009, 9:1451-1468.

2. Andersson L, Porath J: Isolation of phosphoproteins by immobilized metal $\left(\mathrm{Fe}^{3+}\right)$ affinity chromatography. Anal Biochem 1986, 154:250-254

3. Thingholm TE, Jensen ON: Enrichment and characterization of phosphopeptides by immobilized metal affinity chromatography (IMAC) and mass spectrometry. Methods Mol Biol 2009, 527:47-56

4. Pinkse MW, Uitto PM, Hilhorst MJ, Ooms B, Heck AJ: Selective isolation at the femtomole level of phosphopeptides from proteolytic digests using 2D-NanoLC-ESI-MS/MS and titanium oxide precolumns. Anal Chem 2004, 76:3935-3943.

5. Wu J, Shakey Q, Liu W, Schuller A, Follettie MT: Global profiling of phosphopeptides by titania affinity enrichment. J Proteome Res 2007, 6:4684-4689.
6. Villén J, Gygi SP: The SCX/IMAC enrichment approach for global phosphorylation analysis by mass spectrometry. Nat Protoc 2008, 3:1630-1638.

7. Edelmann MJ: Strong cation exchange chromatography in analysis of posttranslational modifications: innovations and perspectives. J Biomed Biotechnol 2011, 2011:936508.

8. Nühse TS, Stensballe A, Jensen ON, Peck SC: Phosphoproteomics of the Arabidopsis plasma membrane and a new phosphorylation site database. Plant Cell 2004, 16:2394-2405.

9. Sugiyama N, Nakagami H, Mochida K, Daudi A, Tomita M, Shirasu K, Ishihama Y: Large-scale phosphorylation mapping reveals the extent of tyrosine phosphorylation in Arabidopsis. Mol Syst Biol 2008, 4:193.

10. Nakagami H, Sugiyama N, Mochida K, Daudi A, Yoshida Y, Toyoda T, Tomita M, Ishihama $Y$, Shirasu K: Large-scale comparative phosphoproteomics identifies conserved phosphorylation sites in plants. Plant Physiol 2010, 153:1161-1174.

11. Meyer $L$, Gao J, Xu D, Thelen JJ: Phosphoproteomic analysis of seed maturation in Arabidopsis, rapeseed, and soybean. Plant Physiol 2012, 159:517-528.

12. Grimsrud PA, den Os D, Wenger CD, Swaney DL, Schwartz D, Sussman MR, Ané J-M, Coon JJ: Large-scale phosphoprotein analysis in Medicago truncatula roots provides insight into in vivo kinase activity in legumes. Plant Physiol 2010, 152:19-28.

13. Rose CM, Venkateshwaran M, Volkening JD, Grimsrud PA, Maeda J, Bailey DJ, Park K, Howes-Podoll M, den Os D, Yeun LH, Westphall MS, Sussman MR, Ané J-M, Coon JJ: Rapid phosphoproteomic and transcriptomic changes in the rhizobia-legume symbiosis. Mol Cell Proteomics 2012, 11:724-744.

14. Bi Y-D, Wang H-X, Lu T-C, X-H L, Shen Z, Chen Y-B, Wang B-C: Large-scale analysis of phosphorylated proteins in maize leaf. Planta 2011, 233:383-392.

15. Facette MR, Shen Z, Björnsdóttir FR, Briggs SP, Smith LG: Parallel proteomic and phosphoproteomic analyses of successive stages of maize leaf development. Plant Cell 2013, 25:2798-2812.

16. Yang F, Melo-Braga MN, Larsen MR, Joergensen HJL, Palmisano G: Battle through signaling between wheat and the fungal pathogen Septoria tritici revealed by proteomics and phosphoproteomics. Mol Cell Proteomics 2013, 12:2497-2508.

17. Lv DW, Ge P, Zhang M, Cheng ZW, Li XH, Yan YM: Integrative network analysis of the signaling cascades in seedling leaves of bread wheat by large-scale phosphoproteomic profiling. J Proteome Res 2014, 13:2381-2395

18. International Brachypodium Initiative: Genome sequencing and analysis of the model grass Brachypodium distachyon. Nature 2010, 463:763-768.

19. Draper J, Mur LA, Jenkins G, Ghosh-Biswas GC, Bablak P, Hasterok R, Routledge AP: Brachypodium distachyon. A new model system for functional genomics in grasses. Plant Physiol 2001, 127:1539-1555.

20. Vogel J, Hill T: High-efficiency Agrobacterium-mediated transformation of Brachypodium distachyon inbred line Bd21-3. Plant Cell Rep 2008, 27:471-478.

21. Volkenburgh EV: Leaf expansion-an integrating plant behaviour. Plant Cell Environ 1999, 22:1463-1473.

22. Munns R, Passioura JB, Guo J, Chazen O, Cramer GR: Water relations and leaf expansion: importance of time scale. J Exp Bot 2000, 51:1495-1504.

23. Pantin F, Simonneau T, Rolland G, Dauzat M, Muller B: Control of leaf expansion: a developmental switch from metabolics to hydraulics. Plant Physiol 2011, 156:803-815.

24. Douché T, Clemente HS, Burlat V, Roujol D, Valot B, Zivy M, Pont-Lezica R, Jamet E: Brachypodium distachyon as a model plant towards improved biofuel crops: search for secreted proteins involved in biogenesis and disassembly of cell wall polymers. Proteomics 2013, 13:2438-2454.

25. Cosgrove DJ: Loosening of plant cell walls by expansins. Nature 2000, 407:321-326

26. He Z-H, Fujiki M, Kohorn BD: A cell wall-associated, receptor-like protein kinase. J Biol Chem 1996, 271:19789-19793.

27. Wagner TA, Kohorn BD: Wall-associated kinases are expressed throughout plant development and are required for cell expansion. Plant Cell 2001, 13:303-318

28. Verica JA, He Z-H: The cell wall-associated kinase (WAK) and WAK-like kinase gene family. Plant Physiol 2002, 129:455-459.

29. Sánchez-Rodríguez C, Estévez JM, Llorente F, Hernández-Blanco C, Jordá L, Pagán I, Berrocal M, Marco Y, Somerville S, Molina A: The ERECTA receptor-like kinase regulates cell wall-mediated resistance to pathogens in Arabidopsis thaliana. Mol Plant Microbe Intera 2009, 22:953-963. 
30. Steinwand BJ, Kieber JJ: The role of receptor-like kinases in regulating cell wall function. Plant Physiol 2010, 153:479-484.

31. Kennelly PJ, Krebs EG: Consensus sequences as substrate specificity determinants for protein kinases and protein phosphatases. J Biol Chem 1991, 266:555-515

32. Kemp BE, Parker MW, Hu S, Tiganis T, House C: Substrate and pseudosubstrate interactions with protein kinases: determinants of specificity. Trends Biochem Sci 1994, 19:440-444.

33. Schwartz D, Gygi SP: An iterative statistical approach to the identification of protein phosphorylation motifs from large-scale data sets. Nat Biotechnol 2005, 23:1391-1398.

34. Schwartz D, Chou MF, Church GM: Predicting protein post-translational modifications using meta-analysis of proteome scale data sets. Mol Cell Proteomics 2009, 8:365-379.

35. Trost M, English L, Lemieux S, Courcelles M, Desjardins M, Thibault P: The phagosomal proteome in interferon- $\gamma$-activated macrophages. Immunity 2009, 30:143-154

36. Bennetzen MV, Larsen DH, Bunkenborg J, Bartek J, Lukas J, Andersen JS: Site-specific phosphorylation dynamics of the nuclear proteome during the DNA damage response. Mol Cell Proteomics 2010, 9:1314-1323.

37. Lv DW, Subburaj S, Cao M, Yan X, Li XH, Appels R, Sun DF, Ma WJ, Yan YM: Proteome and phosphoproteome characterization reveals new response and defense mechanisms of Brachypodium distachyon leaves under salt stress. Mol Cell Proteomics 2014, 13:632-652.

38. Vizcaíno JA, Côté RG, Csordas A, Dianes JA, Fabregat A, Foster JM, Griss J, Alpi E, Birim M, Contell J, O'Kelly G, Schoenegger A, Ovelleiro D, Pérez-Riverol Y, Reisinger F, Ríos D, Wang R, Hermjakob H: The Proteomics Identifications (PRIDE) database and associated tools: status in 2013. Nucleic Acids Res 2013, 41:D1063-D1069.

39. Ghelis T: Signal processing by protein tyrosine phosphorylation in plants. Plant Signal Behav 2011, 6:942-951.

40. Yao Q, Bollinger C, Gao J, Xu D, Thelen JJ: P3DB: an integrated database for plant protein phosphorylation. Front Plant Sci 2012, 3:206.

41. Heazlewood JL, Durek P, Hummel J, Selbig J, Weckwerth W, Walther D, Schulze WX: PhosPhAt: a database of phosphorylation sites in Arabidopsis thaliana and a plant-specific phosphorylation site predictor. Nucleic Acids Res 2008, 36:D1015-D1021

42. Whitmarsh A, Davis R: Regulation of transcription factor function by phosphorylation. Cell Mol Life Sci 2000, 57:1172-1183.

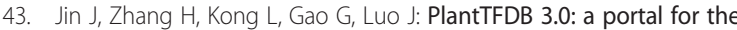
functional and evolutionary study of plant transcription factors. Nucleic Acids Res 2014, 42:D1182-D1187.

44. Arabidopsis Genome Initiative: Analysis of the genome sequence of the flowering plant Arabidopsis thaliana. Nature 2000, 408:796-815.

45. Amanchy R, Periaswamy B, Mathivanan S, Reddy R, Tattikota SG, Pandey A: A curated compendium of phosphorylation motifs. Nat Biotechnol 2007, 25:285-286.

46. Villén J, Beausoleil SA, Gerber SA, Gygi SP: Large-scale phosphorylation analysis of mouse liver. Proc Natl Acad Sci U S A 2007, 104:1488-1493.

47. Prasad TK, Goel R, Kandasamy K, Keerthikumar S, Kumar S, Mathivanan S, Telikicherla D, Raju R, Shafreen B, Venugopal A, Balakrishnan L, Marimuthu A, Banerjee S, Somanathan DS, Sebastian A, Rani S, Ray S, Harrys Kishore CJ, Kanth S, Ahmed M, Kashyap MK, Mohmood R, Ramachandra YL, Krishna V, Rahiman BA, Mohan S, Ranganathan P, Ramabadran S, Chaerkady R, Pandey A: Human protein reference database-2009 update. Nucleic Acids Res 2009, 37:D767-D772

48. Reiland S, Messerli G, Baerenfaller K, Gerrits B, Endler A, Grossmann J, Gruissem W, Baginsky S: Large-scale Arabidopsis phosphoproteome profiling reveals novel chloroplast kinase substrates and phosphorylation networks. Plant Physiol 2009, 150:889-903.

49. Durek P, Schmidt R, Heazlewood JL, Jones A, MacLean D, Nagel A, Kersten B, Schulze WX: PhosPhAt: the Arabidopsis thaliana phosphorylation site database. An update. Nucleic Acids Res 2010, 38:D828-D834.

50. Wang X, Bian Y, Cheng K, Gu LF, Ye M, Zou H, Sun SS, He JX: A large-scale protein phosphorylation analysis reveals novel phosphorylation motifs and phosphoregulatory networks in Arabidopsis. J Proteomics 2013, 78:486-498.

51. Gribskov M, Fana F, Harper J, Hope DA, Harmon AC, Smith DW, Tax FE, Zhang G: PlantsP: a functional genomics database for plant phosphorylation. Nucleic Acids Res 2001, 29:111-113

52. MAPK Group: Mitogen-activated protein kinase cascades in plants: a new nomenclature. Trends Plant Sci 2002, 7:301-308.
53. Ozaki K, Tanaka K, Imamura H, Hihara T, Kameyama T, Nonaka H, Hirano H, Matsuura $Y$, Takai $Y$ : Rom $1 p$ and Rom $2 p$ are GDP/GTP exchange proteins (GEPs) for the Rho1p small GTP binding protein in Saccharomyces cerevisiae. EMBO J 1996, 15:2196-2207.

54. Levin DE: Cell wall integrity signaling in Saccharomyces cerevisiae. Microbiol Mol Biol Rev 2005, 69:262-291.

55. Huang Y, Li H, Gupta R, Morris PC, Luan S, Kieber JJ: ATMPK4, an Arabidopsis homolog of mitogen-activated protein kinase, is activated in vitro by AtMEK1 through threonine phosphorylation. Plant Physiol 2000, 122:1301-1310.

56. Nuhse TS, Peck SC, Hirt H, Boller T: Microbial elicitors induce activation and dual phosphorylation of the Arabidopsis thaliana MAPK 6. Sci Signal 2000, 275:7521-7526.

57. Mironov V, De Veylder L, Van Montagu M, Inzé D: Cyclin-dependent kinases and cell division in plants-the nexus. Plant Cell 1999, 11:509-521.

58. Harmon AC, Gribskov M, Harper JF: CDPKs-a kinase for every $\mathrm{Ca}^{2+}$ signal? Trends Plant Sci 2000, 5:154-159.

59. Ludwig AA, Romeis T, Jones JD: CDPK-mediated signalling pathways: specificity and cross-talk. J Exp Bot 2004, 55:181-188.

60. Tajima Y, Imamura A, Kiba T, Amano Y, Yamashino T, Mizuno T: Comparative studies on the type-B response regulators revealing their distinctive properties in the His-to-Asp phosphorelay signal transduction of Arabidopsis thaliana. Plant Cell Physiol 2004, 45:28-39.

61. Chandrasekher G, Sailaja D: Differential activation of phosphatidylinositol 3-kinase signaling during proliferation and differentiation of lens epithelial cells. Invest Ophthalmol Vis Sci 2003, 44:4400-4411.

62. Carpita NC, Defernez M, Findlay K, Wells B, Shoue DA, Catchpole G, Wilson $\mathrm{RH}, \mathrm{McCann} \mathrm{MC}$ : Cell wall architecture of the elongating maize coleoptile. Plant Physiol 2001, 127:551-565.

63. Fincher GB: Revolutionary times in our understanding of cell wall biosynthesis and remodeling in the grasses. Plant Physio/ 2009 149:27-37.

64. Christensen U, Alonso-Simon A, Scheller HV, Willats WG, Harholt J: Characterization of the primary cell walls of seedlings of Brachypodium distachyon-A potential model plant for temperate grasses. Phytochem 2010, 71:62-69.

65. Hématy K, Sado P-E, Van Tuinen A, Rochange S, Desnos T, Balzergue S, Pelletier $S$, Renou J-P, Höfte H: A receptor-like kinase mediates the response of Arabidopsis cells to the inhibition of cellulose synthesis. Curr Biol 2007, 17:922-931.

66. He ZH, He D, Kohorn BD: Requirement for the induced expression of a cell wall associated receptor kinase for survival during the pathogen response. Plant J 1998, 14:55-63.

67. Lally D, Ingmire $\mathrm{P}$, Tong $\mathrm{H}-\mathrm{Y}, \mathrm{He} Z \mathrm{Z}-\mathrm{H}$ : Antisense expression of a cell wall-associated protein kinase, WAK4, inhibits cell elongation and alters morphology. Plant Cell 2001, 13:1317-1332.

68. He Z-H, Cheeseman I, He D, Kohorn BD: A cluster of five cell wall-associated receptor kinase genes, Wak1-5, are expressed in specific organs of Arabidopsis. Plant Mol Biol 1999, 39:1189-1196.

69. Carpita NC: Structure and biogenesis of the cell walls of grasses. Annu Rev Plant Biol 1996, 47:445-476.

70. Smith BG, Harris PJ: The polysaccharide composition of Poales cell walls: poaceae cell walls are not unique. Biochem Syst Ecol 1999, 27:33-53.

71. Yokoyama R, Nishitani K: Genomic basis for cell-wall diversity in plants. A comparative approach to gene families in rice and Arabidopsis. Plant Cell Physiol 2004, 45:1111-1121

72. Fagard M, Desnos T, Desprez T, Goubet F, Refregier G, Mouille G, McCann M, Rayon C, Vernhettes S, Höfte H: PROCUSTE1 encodes a cellulose synthase required for normal cell elongation specifically in roots and dark-grown hypocotyls of Arabidopsis. Plant Cell 2000, 12:2409-2423.

73. Scheible W-R, Eshed R, Richmond T, Delmer D, Somerville C: Modifications of cellulose synthase confer resistance to isoxaben and thiazolidinone herbicides in Arabidopsis Ixr1 mutants. Proc Natl Acad Sci U S A 2001 98:10079-10084.

74. Somerville C, Bauer S, Brininstool G, Facette M, Hamann T, Milne J, Osborne E, Paredez A, Persson S, Raab T, Vorwerk S, Youngs H: Toward a systems approach to understanding plant cell walls. Sci Signal 2004, 306:2206-2211.

75. Desprez T, Juraniec M, Crowell EF, Jouy H, Pochylova Z, Parcy F, Höfte H, Gonneau M, Vernhettes S: Organization of cellulose synthase complexes involved in primary cell wall synthesis in Arabidopsis thaliana. Proc Natl Acad Sci U S A 2007, 104:15572-15577. 
76. Taylor NG, Howells RM, Huttly AK, Vickers K, Turner SR: Interactions among three distinct CesA proteins essential for cellulose synthesis. Proc Natl Acad Sci U S A 2003, 100:1450-1455.

77. Brown DM, Zeef LA, Ellis J, Goodacre R, Turner SR: Identification of novel genes in Arabidopsis involved in secondary cell wall formation using expression profiling and reverse genetics. Plant Cell 2005, 17:2281-2295.

78. Chu Z, Chen H, Zhang Y, Zhang Z, Zheng N, Yin B, Yan H, Zhu L, Zhao X, Yuan M, Zhang X, Xie Q: Knockout of the AtCESA2 gene affects microtubule orientation and causes abnormal cell expansion in Arabidopsis. Plant Physiol 2007, 143:213-224.

79. Richmond T: Higher plant cellulose synthases. Genome Biol 2000, 1:3001.1-3001.6

80. Freemont PS: Ubiquitination: RING for destruction? Curr Bio/ 2000, 10:R84-R87

81. Kurek I, Kawagoe Y, Jacob-Wilk D, Doblin M, Delmer D: Dimerization of cotton fiber cellulose synthase catalytic subunits occurs via oxidation of the zinc-binding domains. Proc Natl Acad Sci U S A 2002, 99:11109-11114.

82. Chen S, Ehrhardt DW, Somerville CR: Mutations of cellulose synthase (CESA1) phosphorylation sites modulate anisotropic cell expansion and bidirectional mobility of cellulose synthase. Proc Natl Acad Sci U S A 2010, 107:17188-17193.

83. Taylor NG: Identification of cellulose synthase AtCesA7 (IRX3) in vivo phosphorylation sites-a potential role in regulating protein degradation. Plant Mol Biol 2007, 64:161-171.

84. Burton RA, Jobling SA, Harvey AJ, Shirley NJ, Mather DE, Bacic A, Fincher GB: The genetics and transcriptional profiles of the cellulose synthase-like HvCsIF gene family in barley. Plant Physiol 2008, 146:1821-1833.

85. Doblin MS, Pettolino FA, Wilson SM, Campbell R, Burton RA, Fincher GB, Newbigin E, Bacic A: A barley cellulose synthase-like CSLH gene mediates $(1,3 ; 1,4)-\beta-D-g l u c a n$ synthesis in transgenic Arabidopsis. Proc Natl Acad Sci U S A 2009, 106:5996-6001.

86. Gao L, Yan X, Li X, Guo G, Hu Y, Ma W, Yan Y: Proteome analysis of wheat leaf under salt stress by two-dimensional difference gel electrophoresis (2D-DIGE). Phytochemistry 2011, 72:1180-1191.

87. Wang W, Scali M, Vignani R, Spadafora A, Sensi E, Mazzuca S, Cresti M: Protein extraction for two-dimensional electrophoresis from olive leaf, a plant tissue containing high levels of interfering compounds. Electrophoresis 2003, 24:2369-2375.

88. Olsen JV, Blagoev B, Gnad F, Macek B, Kumar C, Mortensen P, Mann M: Global, in vivo, and site-specific phosphorylation dynamics in signaling networks. Cell 2006, 127:635-648.

89. Cox J, Mann M: MaxQuant enables high peptide identification rates, individualized ppb-range mass accuracies and proteome-wide protein quantification. Nat Biotechnol 2008, 26:1367-1372.

90. Conesa A, Gotz S: Blast2GO: A comprehensive suite for functional analysis in plant genomics. Int J Plant Genomics 2008, 2008:619832.

91. Du Z, Zhou X, Ling Y, Zhang Z, Su Z: agriGO: a GO analysis toolkit for the agricultural community. Nucleic Acids Res 2010, 38:W64-W70.

92. Punta M, Coggill PC, Eberhardt RY, Mistry J, Tate J, Boursnell C, Pang N, Forslund K, Ceric G, Clements J, Heger A, Holm L, Sonnhammer EL, Eddy SR, Bateman A, Finn RD: The Pfam protein families database. Nucleic Acids Res 2012, 40:D290-D301

93. Franceschini A, Szklarczyk D, Frankild S, Kuhn M, Simonovic M, Roth A, Lin J, Minguez P, Bork P, von Mering C, Jensen LJ: STRING v9. 1: protein-protein interaction networks, with increased coverage and integration. Nucleic Acids Res 2013, 41:D808-D815.

94. Cline MS, Smoot M, Cerami E, Kuchinsky A, Landys N, Workman C, Christmas R, Avila-Campilo I, Creech M, Gross B, Hanspers K, Isserlin R, Kelley R, Killcoyne S, Lotia S, Maere S, Morris J, Ono K, Pavlovic V, Pico AR, Vailaya A, Wang PL, Adler A, Conklin BR, Hood L, Kuiper M, Sander C, Schmulevich I, Schwikowski B, Warner GJ, et al: Integration of biological networks and gene expression data using Cytoscape. Nat Protoc 2007, 2:2366-2382.

95. Tusnady GE, Simon I: The HMMTOP transmembrane topology prediction server. Bioinformatics 2001, 17:849-850.

doi:10.1186/1471-2164-15-375

Cite this article as: Lv et al:: Large-scale phosphoproteome analysis in seedling leaves of Brachypodium distachyon L. BMC Genomics 2014 15:375.

\section{Submit your next manuscript to BioMed Central and take full advantage of:}

- Convenient online submission

- Thorough peer review

- No space constraints or color figure charges

- Immediate publication on acceptance

- Inclusion in PubMed, CAS, Scopus and Google Scholar

- Research which is freely available for redistribution 